\title{
ON THE SIZE AND SMOOTHNESS OF SOLUTIONS TO NONLINEAR HYPERBOLIC CONSERVATION LAWS*
}

\author{
RONALD A. DEVORE ${ }^{\dagger}$ AND BRADLEY J. LUCIER ${ }^{\ddagger}$
}

\begin{abstract}
We address the question of which function spaces are invariant under the action of scalar conservation laws in one and several space dimensions. We establish two types of results. The first result shows that if the initial data is in a rearrangement-invariant function space, then the solution is in the same space for all time. Secondly, we examine which smoothness spaces among the Besov spaces are invariant for conservation laws. Previously, we showed in one dimension that if the initial data has bounded variation and the flux is convex and smooth enough, then the Besov spaces $B_{q}^{\alpha}\left(L_{q}\right), \alpha>1, q=1 /(\alpha+1)$, are invariant smoothness spaces. Now, in one space dimension, we show that no other Besov space with $\alpha>1$ is invariant. In several space dimensions, we show that no Besov space $B_{q}^{\alpha}\left(L_{q}\right)$ with $\alpha>1$ is invariant. Combined with previous results, our theorems completely characterize for $\alpha>1$ which Besov spaces are smoothness spaces for scalar conservation laws.
\end{abstract}

Key words. Conservation laws, regularity, rearrangement invariant spaces, Besov spaces

AMS subject classifications. 36L65, 35B65, 35D10, 46E30, 46E 35

1. Introduction. We are interested in the size and smoothness, as measured in certain function spaces, of solutions $u(x, t), x \in \mathbb{R}^{d}, t \geq 0$, to the scalar hyperbolic conservation law

$$
\begin{array}{ll}
u_{t}+\nabla_{x} \cdot f(u)=0, & x \in \mathbb{R}^{d}, \quad t>0, \\
u(x, 0)=u_{0}(x), & x \in \mathbb{R}^{d} .
\end{array}
$$

Here, the flux $f$ maps $\mathbb{R}$ into $\mathbb{R}^{d}$ and $\nabla_{x} \cdot f(u)$ denotes the divergence of $f(u(x, t))$ with respect to the spatial variables $x \in \mathbb{R}^{d}$. In general, classical solutions to (1.1) do not exist for all time $t>0$; indeed, at some time $t>0$, which depends on $f$ and $u_{0}$, the solution $u$ to (1.1) will generally develop discontinuities known as "shocks" even if the flux and the initial condition are smooth. One defines weak solutions to (1.1) as functions $u(x, t)$ that satisfy

$$
\begin{aligned}
-\int_{0}^{t} \int_{\mathbb{R}^{d}}\left[u(x, t) \phi_{t}(x, t)+f(u(x, t))\right. & \left.\cdot \nabla_{x} \phi(x, t)\right] d x d t \\
& +\int_{\mathbb{R}^{d}} u(x, T) \phi(x, T) d x-\int_{\mathbb{R}^{d}} u(x, 0) \phi(x, 0) d x=0 .
\end{aligned}
$$

for all $\phi \in C^{1}\left(\mathbb{R}^{d+1}\right)$ with compact support. Weak solutions are not unique; however, by imposing restrictions, known as entropy conditions, on weak solutions $u$, it is possible to select from these weak solutions the physically relevant solution to (1.1). See [10], [15]. When we speak about the solution to (1.1) we shall mean this entropy solution.

* Received by the editors April 29, 1994; accepted for publication September 21, 1994. This research was supported by Office of Naval Research contracts N00014-91-J-1076 and N00014-91-J1152.

† Department of Mathematics, University of South Carolina, Columbia, SC 29208 (devore@math.scarolina.edu).

$\ddagger$ Department of Mathematics, Purdue University, West Lafayet te, IN 47907-1395 (lucier@math.purdue.edu). 
We are interested in the question of which function spaces $X$, such as the spaces of $p$-integrable functions $L_{p}\left(\mathbb{R}^{d}\right)$ or the space of functions of bounded variation $\mathrm{BV}\left(\mathbb{R}^{d}\right)$, are invariant under the differential equation. This means that if we denote the mapping $u_{0} \mapsto u(\cdot, t)$ for fixed $t>0$ by $E(t)$ (i.e., $E(t) u_{0}=u(\cdot, t)$ ), then we are interested in function spaces $X$ for which there exists a constant $C$ such that for for all $u_{0} \in X$

$$
\left\|E(t) u_{0}\right\|_{X} \leq C\left\|u_{0}\right\|_{X} .
$$

If the $X$ norm (or quasi-norm, or semi-norm) in some sense measures smoothness of functions, then we call $X$ a regularity space for (1.1).

We first address the question of how to measure the size of solutions to (1.1). For all convex functions $\eta: \mathbb{R} \rightarrow \mathbb{R}$ and under suitable conditions on $f$, entropy solutions $u(x, t)$ of $(1.1)$ satisfy

$$
\int_{\mathbb{R}^{d}} \eta(u(x, t)) d x \leq \int_{\mathbb{R}^{d}} \eta\left(u_{0}(x)\right) d x
$$

for all $t>0$; see, e.g., [11]. By setting $\eta(u)=|u|^{p}$ for $1 \leq p<\infty$, one sees immediately that

$$
\|u(\cdot, t)\|_{L_{p}\left(\mathbb{R}^{d}\right)} \leq\left\|u_{0}\right\|_{L_{p}\left(\mathbb{R}^{d}\right)} .
$$

One can show independently that (1.2) holds also for $p=\infty$. Thus, $L_{p}\left(\mathbb{R}^{d}\right), 1 \leq$ $p \leq \infty$, are invariant spaces for solutions $u(x, t)$ of (1.1). Actually, one can prove somewhat more, as we now discuss.

The solution operator $E(t)$ of $(1.1)$ is not only bounded on $L_{1}\left(\mathbb{R}^{d}\right)$, but is a contraction in $L_{1}\left(\mathbb{R}^{d}\right)$; i.e., if $u_{0}$ and $v_{0}$ are two initial conditions for (1.1), then

$$
\|u(\cdot, t)-v(\cdot, t)\|_{L_{1}\left(\mathbb{R}^{d}\right)} \leq\left\|u_{0}-v_{0}\right\|_{L_{1}\left(\mathbb{R}^{d}\right)}, \quad t>0 .
$$

Thus, the nonlinear mapping $E(t)$ is a contraction on $X:=L_{1}\left(\mathbb{R}^{d}\right)$ and is bounded on $Y:=L_{\infty}\left(\mathbb{R}^{d}\right)$. A simple argument, given in $\S 4$, shows that if a possibly nonlinear mapping $E(t)$ defined on $X+Y:=\{f+g \mid f \in X, g \in Y\}$ is a contraction on $X$ and is bounded on $Y$, then $E(t)$ is a bounded mapping on all interpolation spaces between $X$ and $Y$ as determined by the method of real interpolation. First results on conservation laws show that $E(t) u_{0}$ is defined for each locally integrable function $u_{0}$, and, in particular, for each function $u_{0}$ in $L_{1}\left(\mathbb{R}^{d}\right)+L_{\infty}\left(\mathbb{R}^{d}\right)$, if $f$ is globally Lipschitz continuous. In our case, this means that $E(t)$ is bounded on all interpolation spaces between $L_{1}\left(\mathbb{R}^{d}\right)$ and $L_{\infty}\left(\mathbb{R}^{d}\right)$, and, in particular on $L_{p}\left(\mathbb{R}^{d}\right)$ for $1<p<\infty$. In addition, many other spaces, such as the Lorentz spaces $L_{p, q}\left(\mathbb{R}^{d}\right), 1 \leq p \leq \infty, 1<q \leq \infty$, and the Orlicz spaces defined on $\mathbb{R}^{d}$, are interpolation spaces for $L_{1}\left(\mathbb{R}^{d}\right)$ and $L_{\infty}\left(\mathbb{R}^{d}\right)$. Calderón [2] characterized the interpolation spaces between $L_{1}\left(\mathbb{R}^{d}\right)$ and $L_{\infty}\left(\mathbb{R}^{d}\right)$ as the set of all rearrangement-invariant function spaces on $\mathbb{R}^{d}$; see $\S 4$. Thus, solutions of (1.1) are bounded on all rearrangement invariant function spaces on $\mathbb{R}^{d}$.

The main focus of this paper is the smoothness, or regularity, of solutions to (1.1), which we next describe. Because of the appearance of shocks, $E(t)$ does not map $C\left(\mathbb{R}^{d}\right)$ into itself. The question arises whether there is any other sense in which the solution of (1.1) retains smoothness.

One should note that solutions of (1.1) are translation invariant, i.e., $E(t)\left(u_{0}\right)(x+$ $h)=E(t)\left(u_{0}(\cdot+h)\right)(x)$ for all $x$ and $h$ in $\mathbb{R}^{d}$. Thus, from (1.3) we see that for all $h \in \mathbb{R}^{d}$,

$$
\|u(\cdot+h, t)-u(\cdot, t)\|_{L_{1}\left(\mathbb{R}^{d}\right)} \leq\left\|u_{0}(\cdot+h)-u_{0}\right\|_{L_{1}\left(\mathbb{R}^{d}\right)} .
$$


Therefore, with the usual norm for the Lipschitz spaces $\operatorname{Lip}\left(\alpha, L^{p}\left(\mathbb{R}^{d}\right)\right)$ given by

$$
\|v\|_{\operatorname{Lip}\left(\alpha, L^{p}\left(\mathbb{R}^{d}\right)\right)}:=\sup _{0 \neq h \in \mathbb{R}^{d}}|h|^{-\alpha}\|v(\cdot+h)-v\|_{L_{p}\left(\mathbb{R}^{d}\right)}, \quad 0<\alpha \leq 1,0<p \leq \infty,
$$

(1.4) implies immediately that

$$
\|u(\cdot, t)\|_{\operatorname{Lip}\left(\alpha, L^{1}\left(\mathbb{R}^{d}\right)\right)} \leq\left\|u_{0}\right\|_{\operatorname{Lip}\left(\alpha, L^{1}\left(\mathbb{R}^{d}\right)\right)}
$$

for all $0<\alpha \leq 1$. In particular, the set of functions of bounded variation on $\mathbb{R}^{d}$, $\mathrm{BV}\left(\mathbb{R}^{d}\right)$, is invariant under $E(t)$, since, by definition, $\operatorname{Lip}\left(1, L^{1}\left(\mathbb{R}^{d}\right)\right)=\mathrm{BV}\left(\mathbb{R}^{d}\right)$.

The Lipschitz spaces $\operatorname{Lip}\left(\alpha, L^{p}\left(\mathbb{R}^{d}\right)\right), 0<\alpha<1$, are special cases of the more general Besov spaces $B_{q}^{\alpha}\left(L_{p}\left(\mathbb{R}^{d}\right)\right.$ ) (see $\S 3$ for a definition), which depend on three parameters $0<\alpha<\infty, 0<p \leq \infty$, and $0<q \leq \infty$. In fact, $\operatorname{Lip}\left(\alpha, L^{p}\left(\mathbb{R}^{d}\right)\right)=$ $B_{\infty}^{\alpha}\left(L_{p}\left(\mathbb{R}^{d}\right)\right)$ for $0<\alpha<1$ and $0<p \leq \infty$. For a Besov space, the parameter $\alpha$ determines the order of smoothness (roughly speaking, the number of derivatives). The second parameter $p$ specifies the space in which smoothness is measured, namely $L_{p}\left(\mathbb{R}^{d}\right)$. The third parameter $q$ allows one to make subtle distinctions in smoothness. Of special interest are the spaces $B_{p}^{\alpha}:=B_{p}^{\alpha}\left(L_{p}\left(\mathbb{R}^{d}\right)\right)$. These are sometimes called fractional order Sobolev spaces because of their similarity to the classical Sobolev spaces; in fact, $B_{2}^{r}$ is identical to the Sobolev space $W_{2}^{r}, r=1,2, \ldots$, consisting of functions from $L_{2}\left(\mathbb{R}^{d}\right)$ that have all of their $r$-th distributional derivatives in $L_{2}\left(\mathbb{R}^{d}\right)$.

The main interest of the present paper is the classification of all Besov spaces $X=B_{p}^{\alpha}, 0<p \leq \infty, 0<\alpha<\infty$, for which $u_{0} \in X$ implies $u(\cdot, t) \in X$ for all later time $t>0$. We shall determine all the regularity spaces of conservation laws among these Besov spaces except for a certain set of values of $\alpha$ and $p$ (with $0 \leq \alpha \leq 1$ ). We use the remainder of this introduction to formulate and explain our results.

We have noted that $\operatorname{Lip}\left(\alpha, L^{1}\left(\mathbb{R}^{d}\right)\right), 0<\alpha \leq 1$, is a regularity space for (1.1) with constant $C=1$. Once one knows the definitions, it is easily shown that all the Besov spaces $B_{q}^{\alpha}\left(L_{1}\left(\mathbb{R}^{d}\right)\right), 0<\alpha<1,0<q \leq \infty$, are regularity spaces, again with $C=1$.

Perhaps somewhat more surprising is the fact established in [6], [7], and [13] that the spaces $\mathrm{BV} \cap B_{\tau(\alpha)}^{\alpha}, \tau(\alpha):=(\alpha+1)^{-1}, \alpha>0$, are regularity spaces in one space dimension provided that the flux $f$ is suitably smooth. In some cases, for example for the inviscid Burgers equation, the space $B_{\tau(\alpha)}^{\alpha}$ is itself a regularity space (i.e., it is not necessary to intersect this space with $\mathrm{BV}$ ).

With these results in hand, the question arises whether any other Besov spaces are regularity spaces for (1.1), to which we now attend. To explain the results of the present paper, it is useful to give a diagram that organizes our knowledge of smoothness spaces. We identify any smoothness space with smoothness $\alpha$ in $L_{p}\left(\mathbb{R}^{d}\right)$, and in particular the Besov space $B_{p}^{\alpha}$, with the point $(1 / p, \alpha)$ in the upper-right quadrant of $\mathbb{R}^{2}$. The classification of Besov spaces as regularity spaces in one space dimension can then be visualized as in Figure 1 . The line segment connecting $(0,0)$ to $(1,0)$ represents the $L_{p}(\mathbb{R})$ spaces, or more generally the rearrangement-invariant spaces (which we show in $\S 4$ are invariant spaces for $(1.1))$. The line segment with endpoints $(1,0)$ and $(1,1)$ represents the regularity spaces $\operatorname{Lip}\left(\alpha, L^{1}(\mathbb{R})\right)$ or $B_{q}^{\alpha}\left(L_{1}\left(\mathbb{R}^{d}\right)\right), 0<\alpha<1$, $0<q \leq \infty$. The half-line $\mathcal{L}_{1}$ with slope one emanating from $(1,0)$ represents the regularity spaces $B_{\tau}^{\alpha}, \tau=\tau(\alpha)=(\alpha+1)^{-1}$ already discussed. Each space to the right of the line $\mathcal{L}_{1}$ contains some functions that are not locally integrable and hence the conservation law does not have a solution for all initial values from these spaces (i.e., the regularity question does not have a meaning). The line $\mathcal{L}_{0}$ emanating from the origin with slope one separates spaces embedded in $C(\mathbb{R})$ (those above the line) 


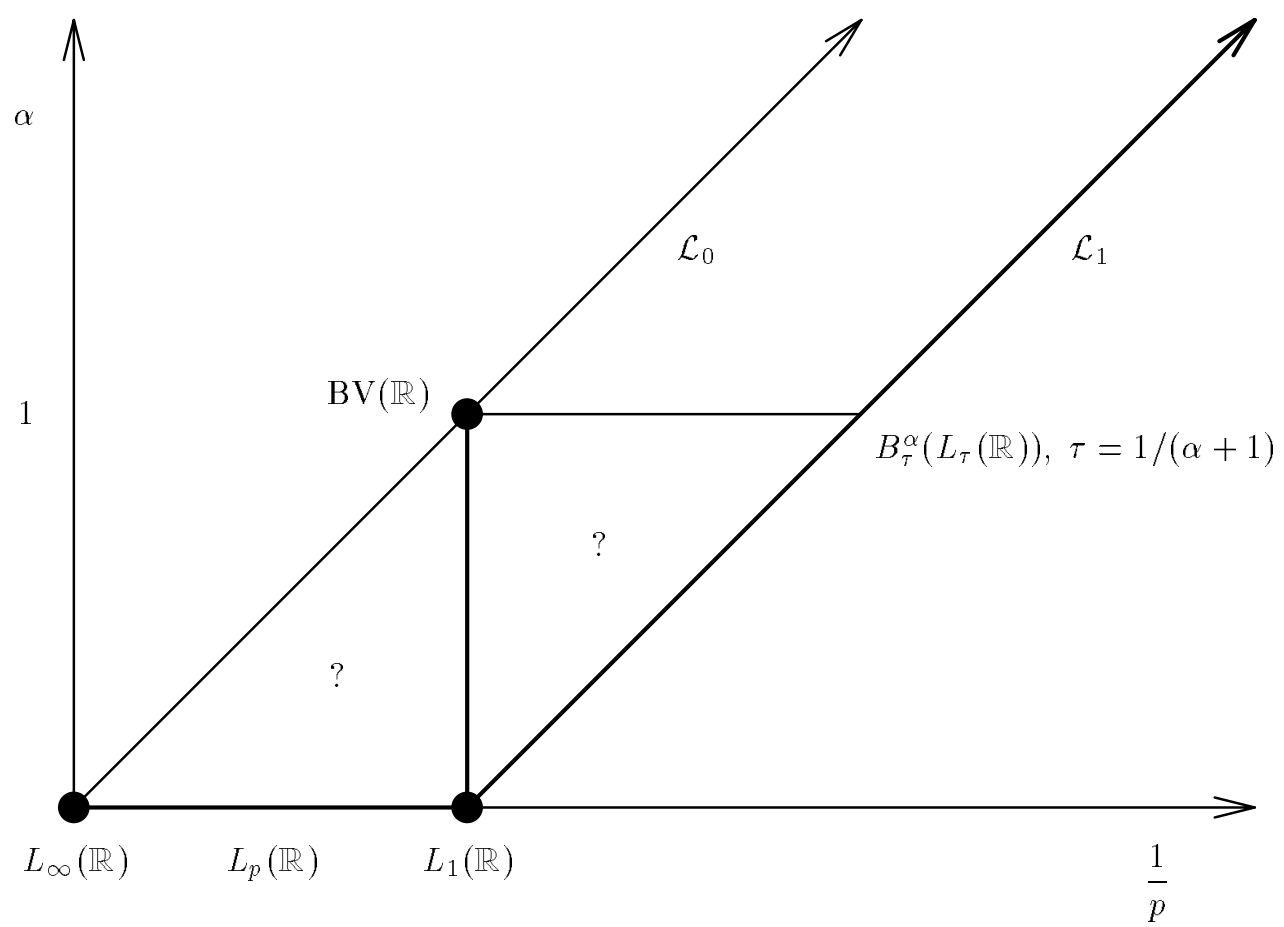

Fig. 1. Smoothness spaces in one space dimension. The line segment from $(1,0)$ to $(1,1)$ represents the spaces $B_{q}^{\alpha}\left(L_{1}(\mathbb{R})\right), 0<\alpha<1,0<q \leq \infty$. The Besov spaces in the open regions not marked by question marks cannot be regularity spaces for (1.1).

and those not embedded in $C(\mathbb{R})$ (below the line). It follows that any space above the line $\mathcal{L}_{0}$ cannot be a regularity space for (1.1), since in general, continuous data $u_{0}$ generate solutions with shocks. We prove in the present paper that for $\alpha>1$, none of the Besov spaces $B_{p}^{\alpha}\left(L_{p}(\mathbb{R})\right)$ are regularity spaces except for the spaces $B_{\tau(\alpha)}^{\alpha}$, which corresponds to points on $\mathcal{L}_{1}$.

In one space dimension, we have not determined whether the spaces represented by points in the parallelogram with vertices $(0,0),(1,0),(1,1)$, and $(2,1)$ are regularity spaces for (1.1). All the same, we conjecture that all these spaces are regularity spaces for (1.1). We are able to use techniques from approximation theory and interpolation of operators to prove this for certain points in this region but will not report on this here since the results are not complete and the arguments are quite technical. One would hope that some nonlinear interpolation argument would settle all these cases.

Our results concerning regularity in one space dimension described above are, for the most part, negative. It is possible, however, to prove some positive results for regularity in one space dimension. We show, using a general argument, that if $f$ is uniformly convex and smooth enough and if $u_{0} \in \mathrm{BV}(\mathbb{R}) \cap B_{\sigma}^{\alpha}\left(L_{\sigma}(\mathbb{R})\right)$ for $\alpha>1$ and $\sigma>\tau(\alpha)=1 /(\alpha+1)$, then for all $t>0, u(\cdot, t)$ is in every Besov space $B_{q}^{\beta}\left(L_{p}(\mathbb{R})\right)$ with $(1 / p, \beta)$ strictly inside the quadrilateral with corners $(0,0),(1,0),(1,1)$, and $(1 / \tau(\alpha), \alpha)$.

The situation regarding regularity spaces in several space dimension is quite different. One might suspect that the Besov spaces $B_{\tau}^{\alpha}, \tau:=(\alpha / d+1)^{-1}$ are regularity spaces for space dimension $d>1$, since their one-dimensional counterparts $(d=1)$ are. (These are precisely the spaces of minimal smoothness that are embedded into 
$L_{1}\left(\mathbb{R}^{d}\right)$, by a variant of the Sobolev embedding theorem.) However, we shall show in this paper that under very general conditions on the flux $f$, none of the Besov spaces $B_{q}^{\alpha}\left(L_{p}\left(\mathbb{R}^{d}\right)\right)$ with $\alpha>1$ are regularity spaces for (1.1). Again, we are not able to completely settle the case $0<\alpha \leq 1$. In $\mathbb{R}^{d}$ the lines $\mathcal{L}_{0}$ and $\mathcal{L}_{1}$ are to be replaced by $\mathcal{L}_{0}^{\prime}$ and $\mathcal{L}_{1}^{\prime}$, which emanate from $(0,0)$ and $(1,0)$, respectively, with slope $d$. We already noted that the spaces on the line segment with endpoints $(1,0),(1,1)$ are regularity spaces.

We feel that our negative results on regularity spaces in several space dimensions give useful information about the structure of solutions to (1.1) and the behavior of numerical methods for their solutions. In order to bring out this point, we first make a few remarks about the connections between regularity and numerical methods. A typical numerical method creates for discrete time values $t_{n}$ an approximation $u_{n}$ to $u\left(\cdot, t_{n}\right)$. The approximants $u_{n}$ will lie in certain linear or nonlinear spaces $\Sigma_{n}$ associated with the numerical method. Usually, $\Sigma_{n}$ is a space of piecewise polynomials with either a fixed or variable grid. The approximation power of such a method is often (although not always) associated with the approximation power from the space $\Sigma_{n}$. In any case, no numerical method can approximate better than the best approximation from $\Sigma_{n}$. The order of best approximation by elements in $\Sigma_{n}$ is characterized by the smoothness of the function $u\left(\cdot, t_{n}\right)$ being approximated. It is therefore important to understand when the solution $u(\cdot, t)$ is in the smoothness space associated with a given order of best approximation by elements in $\Sigma_{n}$. For example, the regularity spaces $B_{\tau(\alpha)}^{\alpha}$ characterize the classes of functions that can be approximated with a given approximation order $N^{-\alpha}$ in $L_{1}(\mathbb{R})$ by free-knot spline functions with $N$ knots. This shows that numerical methods based on moving grids (in one space dimension) should be effective in recovering solutions to scalar conservation laws. This is indeed the case, as is shown in [12], [6], [13] where numerical methods based on moving grid finite elements are constructed that provide approximation order $\alpha$ for any $\alpha>0$.

In the multivariate case, there are no regularity spaces among the Besov spaces $B_{p}^{\alpha}\left(L_{p}\left(\mathbb{R}^{d}\right)\right), \alpha>1$. The Besov spaces are homogeneous: they measure regularity the same way in all coordinate directions. From another viewpoint, these Besov spaces are characterized by very regular approximation processes such as approximation by wavelets or splines with regular partitions. The elements in these spaces behave the same in all coordinate directions. On the other hand, the "fluid transport" in conservation laws can be very directionally dependent. To approximate well such a solution at later time $t>0$ requires finer resolution in directions where mass is accumulating. For example, if we were approximating by piecewise constants we would need elements that are finer in certain directions and coarser in others. This is not possible with splines on regular partitions or wavelets. This is reflected in the fact that their approximation spaces (the Besov spaces) are not regularity spaces for conservation laws in several space dimensions.

2. Properties of entropy solutions to conservation laws. We begin by recalling certain properties of the solution to (1.1) that will be used in the sequel. Let $E(t)$ denote the evolution operator that associates to $u_{0}$ the solution $E(t) u_{0}:=u(\cdot, t)$ of (1.1) at time $t>0$. Then $E(t)$ maps $L_{1}\left(\mathbb{R}^{d}\right)+L_{\infty}\left(\mathbb{R}^{d}\right)$ into itself. Moreover, $E(t)$ is a norm one, bounded operator on $L_{1}\left(\mathbb{R}^{d}\right)$ and $L_{\infty}\left(\mathbb{R}^{d}\right)$ :

$$
\left\|E(t) u_{0}\right\|_{L_{p}\left(\mathbb{R}^{d}\right)} \leq\left\|u_{0}\right\|_{L_{p}\left(\mathbb{R}^{d}\right)}, \quad p=1, \infty .
$$

Actually, $E(t)$ is a bounded operator with norm one on each of the $L_{p}\left(\mathbb{R}^{d}\right)$ spaces 
$1 \leq p \leq \infty$. This can be derived from the study of entropy-entropy flux pairs or proved as in $\S 4$.

The operator $E(t)$ is monotone in the sense that $E(t) u_{0} \geq E(t) v_{0}$ whenever $u_{0} \geq v_{0}$ (see for example [3]) and preserves integrals

$$
\int_{\mathbb{R}^{d}} E(t) u_{0} d x=\int_{\mathbb{R}^{d}} u_{0} d x .
$$

From these properties one derives that $E(t)$ is a contraction (see [3])

$$
\left\|E(t)\left(u_{0}\right)-E(t)\left(v_{0}\right)\right\| \leq\left\|u_{0}-v_{0}\right\| .
$$

Here and later the unsubscripted norm $\|\cdot\|$ always denotes the $L_{1}\left(\mathbb{R}^{d}\right)$ norm.

There is no simple description of the solution $u$ of (1.1). However, in one space dimension, the following method of Lax [11] gives a useful analytic method for obtaining $u$. We assume that the flux $f$ is strictly convex. It follows that the transport velocity $a(u):=f^{\prime}(u)$ is strictly increasing on $\mathbb{R}$ and is therefore invertible (under composition of functions) on $\mathbb{R}$. We assume further that the initial condition $u_{0}$ is continuous with compact support. Any initial condition can be approximated to arbitrary accuracy in the $L_{1}(\mathbb{R})$ norm by such functions. Under these conditions, Lax [11] shows that the solution $u(x, t)$ of $(1.1)$ can be described by

$$
u(x, t)=u_{0}(y(x, t))
$$

where $y=y(x, t)$ satisfies

$$
\frac{x-y}{t}=a\left(u_{0}(y)\right)
$$

In general, there are many solutions $y$ to (2.3). The one that satisfies $(2.2)$ is determined as the solution to an extremal problem (cf. Theorem 3.1 in [11]).

Lax establishes various properties of the selection $y(x, t)$. In particular, he shows that for each fixed $t>0$,

$$
y(\cdot, t) \text { is increasing on } \mathbb{R} \text {. }
$$

Shocks occur in the solution to $(1.1)$ at points where $y(\cdot, t)$ discontinuous. This can occur when there is more than one solution $y$ to $(2.3)$ and we jump down from one piece of the graph of $u_{0}$ to another.

3. Besov spaces. In this section we give the definition of Besov spaces and several equivalent norms for these spaces, which will be used in the sequel. For $\alpha>0$ and $0<p, q \leq \infty$, the Besov space $B_{q}^{\alpha}\left(L_{p}\left(\mathbb{R}^{d}\right)\right)$ is a space of functions with smoothness $\alpha$ in $L_{p}$. The secondary parameter $q$ gives a finer gradation of these spaces that is important in many applications.

To describe these spaces, we use the difference operators $\Delta_{h}^{r}, r=1,2, \ldots$, with step $h \in \mathbb{R}^{d}$. These are defined inductively with $\Delta_{h}(v, x):=v(x+h)-v(x)$ and $\Delta_{h}^{r}:=\Delta_{h} \Delta_{h}^{r-1}$. It follows that

$$
\Delta_{h}^{r}(v, x):=\sum_{j=0}^{r}(-1)^{r+j}\left(\begin{array}{l}
r \\
j
\end{array}\right) v(x+j h) .
$$

With these differences, we can define the moduli of smoothness

$$
\omega_{r}(v, s)_{p}:=\sup _{0 \leq|h| \leq s}\left\|\Delta_{h}^{r}(v)\right\|_{L_{p}\left(\mathbb{R}^{d}\right)}, \quad s \geq 0,
$$


for each $r=1,2, \ldots$. The rate at which $\omega_{r}(v, t)_{p}$ tends to zero gives information about the smoothness of $v$ in $L_{p}\left(\mathbb{R}^{d}\right)$.

The Besov spaces are defined for $0<\alpha<r$ and $0<p, q \leq \infty$ as the set of all functions $v \in L_{p}\left(\mathbb{R}^{d}\right)$ for which

$$
|v|_{B_{q}^{\alpha}\left(L_{p}\left(\mathbb{R}^{d}\right)\right)}:= \begin{cases}\left(\int_{0}^{\infty}\left[s^{-\alpha} \omega_{r}(v, s)_{p}\right]^{q} \frac{d s}{s}\right)^{\frac{1}{q}}, & 0<q<\infty, \\ \sup _{s \geq 0} s^{-\alpha} \omega_{r}(v, s)_{p}, & q=\infty,\end{cases}
$$

is finite. The conditions $(3.2)$ require that $\omega_{r}(v, s)_{p}$ behave like $O\left(s^{\alpha}\right)$ as $s \rightarrow 0$; the exact requirement on $\omega_{r}(v, s)_{p}$ varies with $q$ and becomes stronger as $q$ gets smaller. We define the following "norm" for $B_{q}^{\alpha}\left(L_{p}\left(\mathbb{R}^{d}\right)\right)$ :

$$
\|v\|_{B_{q}^{\alpha}\left(L_{p}\left(\mathbb{R}^{d}\right)\right)}:=\|v\|_{L_{p}\left(\mathbb{R}^{d}\right)}+|v|_{B_{q}^{\alpha}\left(L_{p}\left(\mathbb{R}^{d}\right)\right)} .
$$

Because we allow $p$ and $q$ to be less than 1, this "norm" does not always satisfy the triangle inequality, but it is always a quasi-norm, i.e., there exists a constant $C$ such that for all $u$ and $v$ in $B_{q}^{\alpha}\left(L_{p}\left(\mathbb{R}^{d}\right)\right)$,

$$
\|u+v\|_{B_{q}^{\alpha}\left(L_{p}\left(\mathbb{R}^{d}\right)\right)} \leq C\left(\|u\|_{B_{q}^{\alpha}\left(L_{p}\left(\mathbb{R}^{d}\right)\right)}+\|v\|_{B_{q}^{\alpha}\left(L_{p}\left(\mathbb{R}^{d}\right)\right)}\right) .
$$

It can be shown that the above definition of Besov spaces does not depend on the choice of $r$, since all values of $r>\alpha$ give rise to equivalent norms and hence the same space. We note that since $\omega_{r}(v, t)_{p} \leq 2^{r}\|v\|_{L_{p}\left(\mathbb{R}^{d}\right)}, 1 \leq p \leq \infty$ (the same inequality holds with $2^{r}$ replaced by $2^{r / p}$ when $p<1$ ), we obtain an equivalent norm for $B_{q}^{\alpha}\left(L_{p}\left(\mathbb{R}^{d}\right)\right)$ if we take the integral or supremum in (3.2) over only the interval $[0,1]$. Thus, membership of $v$ in $B_{q}^{\alpha}\left(L_{p}\left(\mathbb{R}^{d}\right)\right)$ is determined only by the integral or supremum on $[0,1]$.

For certain values of the parameters, the Besov spaces are identical with other smoothness spaces. For example, if $0<p \leq \infty$ and $0<\alpha$ is not an integer, then $B_{\infty}^{\alpha}\left(L_{p}\left(\mathbb{R}^{d}\right)\right)=\operatorname{Lip}\left(\alpha, L_{p}\left(\mathbb{R}^{d}\right)\right)$ are the classical Lipschitz spaces. When $\alpha=k$ is an integer we obtain the generalized Lipschitz spaces, for which $\omega_{r}(v, s)_{p}, r>k$ is used in place of $\omega_{k}(v, s)_{p}$ in the definition of the usual Lipschitz spaces. For $p=2$ and $\alpha>0$, the Besov spaces $B_{2}^{\alpha}\left(L_{2}\left(\mathbb{R}^{d}\right)\right)=W^{\alpha}\left(L_{2}\left(\mathbb{R}^{d}\right)\right)$ are the Sobolev spaces. The Besov spaces with $q=p$, which we shall denote by $B_{p}^{\alpha}:=B_{p}^{\alpha}\left(L_{p}\left(\mathbb{R}^{d}\right)\right)$, are of particular interest. These are sometimes called generalized Sobolev spaces.

The application of Besov spaces to approximation theory and interpolation of linear operators leads to alternate characterizations of these spaces. We shall mention two of these alternate characterizations that hold in the univariate case.

The first characterization describes the Besov spaces in terms of approximation by linear spaces of spline functions. Let $\mathcal{S}_{n, r}$ denote the set of all univariate piecewise polynomials of degree $<r$ that have global smoothness $C^{(r-2)}$ and have break points only at the dyadic integers $j 2^{-n}, j \in \mathbb{Z}$. For each $f \in L_{p}(\mathbb{R})$, we define

$$
s_{n}(f)_{p}:=s_{n, r}(f)_{p}:=\inf _{S \in \mathcal{S}_{n, r}}\|f-S\|_{L_{p}(\mathbb{R})}
$$

Then, (see [8]), for $0<\alpha<r$, a function $f$ is in $B_{q}^{\alpha}\left(L_{p}(\mathbb{R})\right)$ if and only if

$$
\left(\sum_{n=0}^{\infty}\left[2^{n \alpha} s_{n}(f)_{p}\right]^{q}\right)^{1 / q}<\infty
$$


with the usual change to a supremum when $q=\infty$. Moreover, (3.3) gives an equivalent semi-norm for $B_{q}^{\alpha}\left(L_{p}(\mathbb{R})\right)$. It follows directly from $(3.3)$ that $B_{\sigma}^{\alpha}\left(L_{p}(\mathbb{R})\right)$ is continuously embedded in $B_{\tau}^{\beta}\left(L_{p}(\mathbb{R})\right)$ if $\alpha>\beta$ or $\alpha=\beta$ and $\sigma<\tau$.

Our second characterization describes the Besov spaces in terms of univariate nonlinear approximation. For this, we let $\Sigma_{n}:=\Sigma_{n, r}$ denote the collection of all piecewise polynomial functions of degree $<r$ on $\mathbb{R}$ that consist of at most $n+1$ pieces. Thus $S \in \Sigma_{n}$ if and only if there exist $n$ breakpoints $x_{1}<x_{2}<\cdots<x_{n}$ such that with $x_{0}:=-\infty, x_{n+1}:=\infty$, the function $S$ is a polynomial of degree $<r$ on each of the intervals $\left(x_{i-1}, x_{i}\right), i=1, \ldots, n$. No assumption is made about the smoothness of $S$ at the breakpoints. The set $\Sigma_{n}$ is not a linear space, but it can be considered a nonlinear manifold parameterized by the breakpoints and the coefficients of the polynomial pieces.

We can describe certain Besov spaces in terms of their approximation by the elements of $\Sigma_{n}$. For this, we define for $f \in L_{p}(\mathbb{R}), 0<p \leq \infty$,

$$
\sigma_{n}(f)_{p}:=\sigma_{n, r}(f)_{p}:=\inf _{S \in \Sigma_{n}}\|f-S\|_{L_{p}(\mathbb{R})}
$$

which is the error in approximating $f$ in the $L_{p}(\mathbb{R})$ norm by the elements of $\Sigma_{n}$.

Nonlinear spline approximation can be used to characterize certain of the spaces $B_{\tau}^{\alpha}$ (see [14] and [9]). If $r>\alpha>0$ and $\tau>0$ are given, and if there is a $p$ with $0<p<\infty$ such that $\tau=\tau(\alpha, p):=(\alpha+1 / p)^{-1}$, then $f \in B_{\tau}^{\alpha}\left(L_{\tau}(\mathbb{R})\right)$ if and only if

$$
\left(\sum_{n=1}^{\infty}\left[2^{n \alpha} \sigma_{2^{n}}(f)_{p}\right]^{\tau}\right)^{1 / \tau}<\infty
$$

and (3.5) when added to $\|\cdot\|_{L_{\tau}(\mathbb{R})}$ gives an equivalent norm for $B_{\tau}^{\alpha}$ :

$$
\|f\|_{B_{\tau}^{\alpha}\left(L_{\tau}(\mathbb{R})\right)} \sim\|f\|_{L_{\tau}(\mathbb{R})}+\left(\sum_{n=1}^{\infty}\left[2^{n \alpha} \sigma_{2^{n}}(f)_{p}\right]^{\tau}\right)^{1 / \tau} .
$$

Furthermore, (3.6) implies that $B_{\tau(\alpha, p)}^{\alpha}$ is continuously embedded in $B_{\tau(\beta, p)}^{\beta}$ if $\alpha>\beta$. Indeed, $B_{\tau(\alpha, p)}^{\alpha}$ is continuously embedded in $L_{p}(\mathbb{R})$ (see [8]), and the family of spaces $B_{\tau(\alpha, p)}^{\alpha}$ lies on the half-line with slope one emanating from the point $(1 / p, 0)$ in Figure 1.

It may be useful to say a few words about the differences in the two characterizations (3.3) and (3.5). The characterization (3.3) describes the space $B_{q}^{\alpha}\left(L_{p}\left(\mathbb{R}^{d}\right)\right.$ ) in terms of approximation in $L_{p}\left(\mathbb{R}^{d}\right)$. Thus the approximation is taking place in the same space $\left(L_{p}\left(\mathbb{R}^{d}\right)\right)$ in which the smoothness is measured. In contrast, in (3.5) the approximation takes place in the space $L_{p}\left(\mathbb{R}^{d}\right)$ but the smoothness is measured in the space $L_{\tau}\left(\mathbb{R}^{d}\right)$; this is characteristic of nonlinear approximation. Since $\tau<p$, the class of functions that can be approximated by the nonlinear family $\Sigma_{n}$ is larger than the class that is approximated by the linear spaces $\mathcal{S}_{n}$.

4. Rearrangement invariant spaces. In this section, we shall give an elementary approach to finding invariant spaces based on interpolation of operators. We begin by recalling some basic facts about the $K$-functional and its application to the theory of interpolation of operators.

If $\left(X_{0}, X_{1}\right)$ is a pair of complete, quasi-normed spaces embedded in a Hausdorff 
space $\mathcal{X}$, then for each $v \in X_{0}+X_{1}$, we form the $K$-functional

$$
K(v, s):=K\left(v, s ; X_{0}, X_{1}\right):=\inf _{v=v_{0}+v_{1}}\left\{\left\|v_{0}\right\|_{X_{0}}+s\left\|v_{1}\right\|_{X_{1}}\right\}, \quad s \geq 0,
$$

where the infimum is taken over all decompositions $v=v_{0}+v_{1}$ with $v_{i} \in X_{i}, i=0,1$. It is easy to see that for fixed $s>0, K(\cdot, s)$ is a quasi-norm and for fixed $v, K(v, \cdot)$ is an increasing concave function on $\mathbb{R}_{+}$.

The $K$-functional was introduced by Peetre as a tool for obtaining interpolation spaces $X$ for the pair $\left(X_{0}, X_{1}\right)$. We recall that a complete quasi-normed space $X$ contained in $X_{0}+X_{1}$ is called an interpolation space for the pair $\left(X_{0}, X_{1}\right)$ if each linear operator $T$ that boundedly maps $X_{0}$ and $X_{1}$ into themselves also maps $X$ boundedly into itself. For such a $T$, it follows that

$$
K\left(T v, s ; X_{0}, X_{1}\right) \leq M K\left(v, s ; X_{0}, X_{1}\right), \quad s>0,
$$

with $M$ the maximum of the norm of $T$ on the two spaces $X_{0}, X_{1}$.

In view of (4.1), we can obtain interpolation spaces for $\left(X_{0}, X_{1}\right)$ by applying to $K(v, \cdot)$ a quasi-norm defined for functions on $\mathbb{R}_{+}$. We mention in particular the $(\theta, q)$ norms, $0<\theta<1,0<q \leq \infty$, which give the spaces $X_{\theta, q}$ that consist of all $v \in X_{0}+X_{1}$ for which

$$
|v|_{X_{\theta, q}}:=\left(\int_{0}^{\infty}\left[s^{-\theta} K(v, s)\right]^{q} \frac{d s}{s}\right)^{1 / q}
$$

is finite (with the usual change to a supremum when $q=\infty$ ). It follows from (4.1) that $T$ maps $X_{\theta, q}$ into itself for each $0<\theta<1,0<q \leq \infty$, with a norm not exceeding $M$.

It is not possible to apply this interpolation directly to the operator $E(t)$ associated with (1.1) since it is not linear. However, the following simple remark can be used in place of linearity. We say that an operator $T$ is $X_{0}$-Lipschitz on $X_{0}+X_{1}$ if

$$
\left\|T\left(v_{0}\right)-T\left(v_{1}\right)\right\|_{X_{0}} \leq M_{0}\left\|v_{0}-v_{1}\right\|_{X_{0}}
$$

for each $v_{0}, v_{1} \in X_{0}+X_{1}$ for which $v_{1}-v_{0} \in X_{0}$.

LEMMA 4.1. If $T$ is a (possibly nonlinear) operator that is $X_{0}$-Lipschitz with constant $M_{0}$ on $X_{0}+X_{1}$ and is bounded with norm $M_{1}$ on $X_{1}$, then $T$ satisfies

$$
K(T v, s) \leq M K(v, s), \quad v \in X_{0}+X_{1}, s>0 .
$$

with $M:=\max \left(M_{0}, M_{1}\right)$.

Proof. The proof is almost a triviality. Let $s>0$. For a given $\epsilon>0$, let $v=$ $v_{0}+v_{1}$ be a decomposition of $v$ that satisfies $\left\|v_{0}\right\|_{X_{0}}+s\left\|v_{1}\right\|_{X_{1}} \leq K(v, s)+\epsilon$. Then $T v=\left(T v-T v_{1}\right)+T v_{1}$ and $v-v_{1}=v_{0} \in X_{0}$. Hence,

$$
\left\|T v-T v_{1}\right\|_{X_{0}}+s\left\|T v_{1}\right\|_{X_{1}} \leq M_{0}\left\|v-v_{1}\right\|_{X_{0}}+s M_{1}\left\|v_{1}\right\|_{X_{1}} \leq M(K(f, s)+\epsilon) .
$$

Since $\epsilon>0$ is arbitrary, (4.2) follows from the definition of the $K$-functional.

We now apply this lemma to the solution operator $E:=E(t)$ for the conservation law (1.1). The contractivity of the operator $E$ on $L_{1}\left(\mathbb{R}^{d}\right)$ has a local variant. For this, we assume that the flux $f$ is in Lip 1. It follows that the transport velocity vector $f^{\prime}(u)$ satisfies

$$
\Lambda:=\sup _{u \in \mathbb{R}}\left\|f^{\prime}(u)\right\|_{\ell_{1}}<\infty
$$


where $\|x\|_{\ell_{1}}:=\sum_{i=1}^{d}\left|x_{i}\right|$. Under this assumption, we have ([10]) for any locally integrable functions $u_{0}, v_{0}$ and any ball $B(x, R)$ of radius $R>0$ centered at the point $x$,

$$
\int_{B(x, R)}\left|E\left(u_{0}\right)-E\left(v_{0}\right)\right| \leq \int_{B(x, R+\Lambda t)}\left|u_{0}-v_{0}\right|
$$

In particular, if $u_{0}, v_{0} \in L_{1}\left(\mathbb{R}^{d}\right)+L_{\infty}\left(\mathbb{R}^{d}\right)$ and $u_{0}-v_{0} \in L_{1}\left(\mathbb{R}^{d}\right)$, then taking a limit as $R \rightarrow \infty$ in (4.3) shows that $E$ is $L_{1}$-Lipschitz on $L_{1}+L_{\infty}$ with constant 1 . Since $E$ is a norm-one operator on $L_{\infty}$, we can apply Lemma 4.1 and find

$$
K\left(E\left(u_{0}\right), s ; L_{1}\left(\mathbb{R}^{d}\right), L_{\infty}\left(\mathbb{R}^{d}\right)\right) \leq K\left(u_{0}, s ; L_{1}\left(\mathbb{R}^{d}\right), L_{\infty}\left(\mathbb{R}^{d}\right)\right)
$$

The $K$-functional for the pair $\left(L_{1}\left(\mathbb{R}^{d}\right), L_{\infty}\left(\mathbb{R}^{d}\right)\right)$ can be described in terms of rearrangements of functions. We refer the reader to the book of Bennett and Sharpley [1] and Calderón's paper [2] for a discussion of rearrangements and the material that follows in this section. The rearrangement $v^{*}$ of a function $v \in L_{1}\left(\mathbb{R}^{d}\right)+L_{\infty}\left(\mathbb{R}^{d}\right)$ is a non-increasing function defined on $\mathbb{R}_{+}$that is equimeasurable with $v$, i.e,

$$
\operatorname{meas}\left\{x \in \mathbb{R}^{d}|| v(x) \mid>y\right\}=\operatorname{meas}\left\{x \in \mathbb{R}_{+}|| v^{*}(x) \mid>y\right\}
$$

for all $y \geq 0$. It follows that the rearrangement $v^{*}$ of any $v \in L_{p}\left(\mathbb{R}^{d}\right)$ is in $L_{p}\left(\mathbb{R}_{+}\right)$and

$$
\|v\|_{L_{p}\left(\mathbb{R}^{d}\right)}=\left\|v^{*}\right\|_{L_{p}\left(\mathbb{R}_{+}\right)}, \quad 1 \leq p \leq \infty .
$$

It can readily be verified that the $K$-functional for $\left(L_{1}, L_{\infty}\right)$ is

$$
K\left(v, s ; L_{1}\left(\mathbb{R}^{d}\right), L_{\infty}\left(\mathbb{R}^{d}\right)\right)=\int_{0}^{s} v^{*}(y) d y .
$$

The Hardy-Littlewood maximal function $v^{* *}$ of $v^{*}$ is related to the $K$-functional $K\left(v, s ; L_{1}\left(\mathbb{R}^{d}\right), L_{\infty}\left(\mathbb{R}^{d}\right)\right)$ in the following way:

$$
v^{* *}(s):=\frac{1}{s} \int_{0}^{s} v^{*}(y) d y=\frac{1}{s} K\left(v, s ; L_{1}\left(\mathbb{R}^{d}\right), L_{\infty}\left(\mathbb{R}^{d}\right)\right) .
$$

Using the functions $v^{* *}$, Calderón defines a normed space $X \subset L^{1}\left(\mathbb{R}^{d}\right)+L^{\infty}\left(\mathbb{R}^{d}\right)$ as rearrangement invariant if

$$
f \in X \text { and } g^{* *}(s) \leq f^{* *}(s), s \geq 0, \Longrightarrow g \in X \text { and }\|g\|_{X} \leq\|f\|_{X} .
$$

Calderón showed that the interpolation spaces for the pair $\left(L_{1}\left(\mathbb{R}^{d}\right), L_{\infty}\left(\mathbb{R}^{d}\right)\right)$ consist precisely of the set of rearrangement-invariant spaces.

As a consequence, from Lemma 4.1 we obtain the following.

THEOREM 4.2. If the flux $f$ is in $\operatorname{Lip} 1$, then for any $t>0$, the evolution operator $E=E(t)$ for the conservation law (1.1) when applied to an arbitrary function $u_{0} \in$ $L_{1}\left(\mathbb{R}^{d}\right)+L_{\infty}\left(\mathbb{R}^{d}\right)$ satisfies

$$
E\left(u_{0}\right)^{* *}(s) \leq u_{0}^{* *}(s) \quad s \geq 0 .
$$

In particular, E maps every rearrangement-invariant space $X$ on $\mathbb{R}^{d}$ into itself with norm 1.

Inequality (4.7) gives precise information about the relative sizes of $u_{0}$ and $E u_{0}$. For example, if we let $d=1, f(u)=u^{2}, u_{0}$ equal the characteristic function of $[0,1]$, 

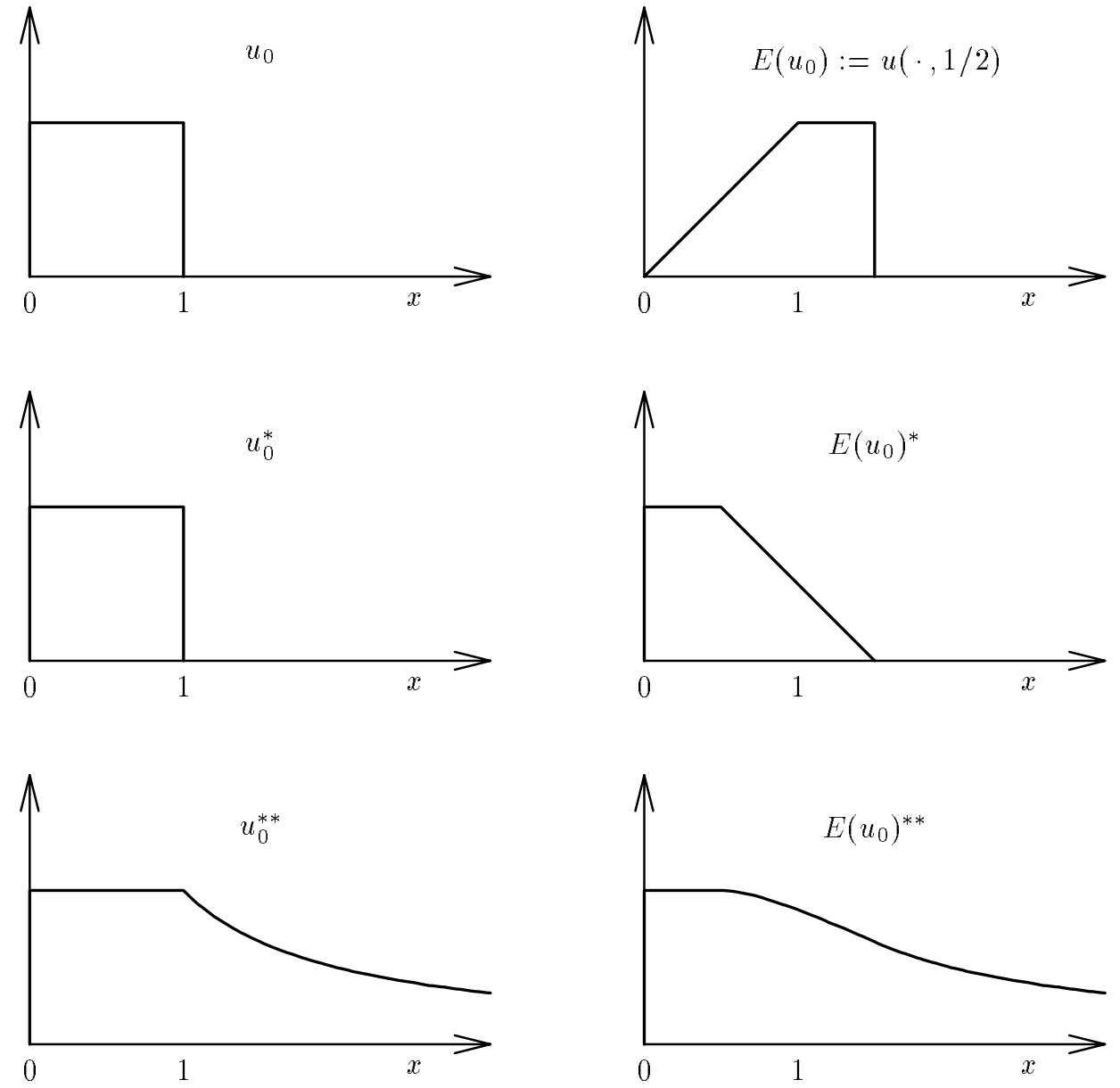

Fig. 2. For these figures, the flux is $f(u)=u^{2}$. The initial data $u_{0}=\chi_{[0,1]}$, its nonincreasing rearrangement $u_{0}^{*}$, and $u_{0}^{* *}$, the maximal function of $u_{0}^{*}$. Similarly for $E\left(u_{0}\right):=u(\cdot, 1 / 2)$.

and $t=1 / 2$, then $u_{0}=u_{0}^{*}$,

$$
E\left(u_{0}\right)(x)=\left\{\begin{array}{ll}
0, & x \leq 0, \\
x, & 0 \leq x \leq 1, \\
1, & 1 \leq x \leq 3 / 2, \\
0, & 1<x .
\end{array} \quad \text { and } \quad E\left(u_{0}\right)^{*}(x)= \begin{cases}0, & x<0, \\
1, & 0 \leq x \leq 1 / 2, \\
3 / 2-x, & 1 / 2 \leq x \leq 3 / 2, \\
0, & 3 / 2 \leq x .\end{cases}\right.
$$

Note that for $1<x<3 / 2, u_{0}^{*}(x)<E\left(u_{0}\right)^{*}(x)$, yet, by Theorem 4.2 (or an easy direct calculation) we have that

$$
E\left(u_{0}\right)^{* *}(x) \leq u_{0}^{* *}(x)
$$

for all $x>0$. See Figure 2 .

Some examples of rearrangement-invariant spaces are the $L_{p}\left(\mathbb{R}^{d}\right)$ spaces, $1 \leq p \leq$ $\infty$, and the Lorentz spaces $L_{p, q}\left(\mathbb{R}^{d}\right), 1 \leq p \leq \infty, 1 \leq q \leq \infty$; for $p>1$, these consist 
of all $v \in L_{1}\left(\mathbb{R}^{d}\right)+L_{\infty}\left(\mathbb{R}^{d}\right)$ for which

$$
\|v\|_{L_{p, q}\left(\mathbb{R}^{d}\right)}:=\left(\int_{0}^{\infty}\left[s^{1 / p} v^{* *}(s)\right]^{q} \frac{d s}{s}\right)^{1 / q}
$$

is finite. Other rearrangement-invariant function spaces include $L \log L$ and Orlicz spaces.

5. Regularity in Besov spaces. Part I: Positive results. The remainder of this paper concerns itself with the regularity of the solution $u(\cdot, t)$ of $(1.1)$ as measured in Besov spaces. We continue to use the notation $\tau(\alpha, p):=(\alpha+1 / p)^{-1}$ and $B_{s}^{\alpha}:=B_{s}^{\alpha}\left(L_{s}\left(\mathbb{R}^{d}\right)\right)$.

It is well known that if the initial data $u_{0}$ is of bounded variation, then the solution $u(\cdot, t)$ of $(1.1)$ is of bounded variation for all positive time $t$ and

$$
|u(\cdot, t)|_{\mathrm{BV}} \leq\left|u_{0}\right|_{\mathrm{BV}}
$$

for all $t>0$. In addition, we showed in one space dimension [6], [13] that if $f \in C^{r+1}$ is globally Lipschitz continuous and uniformly convex, and $u_{0} \in \mathrm{BV} \cap B_{\tau(\alpha, 1)}^{\alpha}$ for some $1<\alpha<r$, then for all $t>0, u(\cdot, t) \in \mathrm{BV} \cap B_{\tau(\alpha, 1)}^{\alpha}$, and

$$
\|u(\cdot, t)\|_{B_{\tau(\alpha, 1)}^{\alpha}} \leq C\left(\left\|u_{0}\right\|_{B_{\tau(\alpha, 1)}^{\alpha}}+1\right)
$$

where $C$ depends only on $r, t,\left\|f^{(r+1)}\right\|_{L_{\infty}}$, and $\left|u_{0}\right|_{\mathrm{BV}}$; i.e., $\mathrm{BV} \cap B_{\tau(\alpha, 1)}^{\alpha}$ is a regularity space for (1.1). (For the inviscid Burgers equation

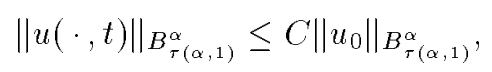

where $C$ depends only on $\alpha$.) One can ask whether any other spaces $B_{q}^{\alpha}\left(L_{\sigma}(\mathbb{R})\right) \cap$ $\mathrm{BV}(\mathbb{R})$ are also regularity spaces for (1.1). As we have explained in the introduction, this cannot hold for Besov spaces that correspond to points above the line $\mathcal{L}_{0}$ or below the line $\mathcal{L}_{1}$ of Figure 1. Thus we can restrict our attention to spaces corresponding to points in the region bounded by $\mathcal{L}_{0}, \mathcal{L}_{1}$, and the $x$-axis. Each Besov space in this region is of the form $B_{q}^{\alpha}\left(L_{\tau(\alpha, p)}\right)$ for some $p$ between 1 and infinity, $0<q \leq \infty$, and $\alpha>0$.

In this section, we show that if $u_{0} \in \mathrm{BV} \cap B_{\tau(\alpha, p)}^{\alpha}, \alpha>1$ and $1 \leq p \leq \infty$, then $u(\cdot, t)$ is in $\mathrm{BV} \cap B_{\tau(\beta, q)}^{\beta}$ for all $1<q<\infty$ and $\beta<1+\frac{1}{q}(\alpha-1)$. The points $(1 / \tau(\beta, q), \beta), \beta=1+\frac{1}{q}(\alpha-1)$, lie on the line segment joining the points $(1,1)$ and $(1 / \tau(\alpha, 1), \alpha)$ in Figure 1; i.e., these spaces are intermediate to $\mathrm{BV}$ and $B_{\tau(\alpha, 1)}^{\alpha}$. In the next two sections we show that, in general, $u(\cdot, t)$ may not be in $\mathrm{BV} \cap B_{\tau(\beta, q)}^{\beta}$ for $1<q<\infty$ whenever $\beta>1+\frac{1}{q}(\alpha-1)$. In particular, we can say that the $B_{\tau(\alpha, p)}^{\alpha}$, $\alpha>1$, are not regularity spaces for (1.1) for any values of $p \neq 1$. As in [7], we can remove the restriction of $\mathrm{BV}$ functions if $f(u)=u^{2}$.

We first prove the following lemma, which holds for general functions $v$, not necessarily solution of (1.1).

Lemma 5.1. If $v \in \mathrm{BV} \cap B_{\tau(\alpha, 1)}^{\alpha}, \alpha>1$, then $v \in B_{\sigma}^{\beta}\left(L_{\tau(\beta, p)}\right)$ for $\beta=1+\frac{1}{p}(\alpha-1)$, $1<p<\infty$, and $\sigma=p \tau(\alpha, 1)$.

Proof. The proof will be based on a standard interpolation argument. We take as the $\mathrm{BV}$-seminorm

$$
|v|_{\mathrm{BV}}=\sup _{s>0} \frac{\omega_{1}(v, s)_{1}}{s} .
$$


Since for any $r \geq 1, \omega_{r}(v, s)_{1} \leq 2^{r-1} \omega_{1}(v, s)_{1}$, we have

$$
\omega_{r}(v, s)_{1} \leq C \omega_{1}(v, s)_{1} \leq C s|v|_{\mathrm{BV}}
$$

with $C$ depending only on $r$.

We now assume that $1<\alpha<r$, and we write $\mu:=\tau(\alpha, 1)$ and $\lambda:=\tau(\beta, p)$, i.e.,

$$
\frac{1}{\mu}=\alpha+1 \text { and } \frac{1}{\lambda}=\beta+\frac{1}{p}=\frac{\alpha}{p}+1 \text {. }
$$

Notice that

$$
\left(\frac{1}{\lambda}, \beta\right)=\frac{1}{p}\left(\frac{1}{\mu}, \alpha\right)+\left(1-\frac{1}{p}\right)(1,1),
$$

that is, $(1 / \lambda, \beta)$ is a convex combination of $(1 / \mu, \alpha)$ and $(1,1)$. We shall estimate $\omega_{r}(v, s)_{\lambda}$. We use the abbreviated notation $\Delta(x):=\left|\Delta_{h}^{r}(v, x)\right|$. We let $p^{\prime}$ be the conjugate exponent to $p$, i.e., $\frac{1}{p}+\frac{1}{p^{\prime}}=1$. It follows from Hölder's inequality that

$$
\begin{aligned}
\int_{\mathbb{R}} \Delta(x)^{\lambda} d x & =\int_{\mathbb{R}} \Delta(x)^{\frac{\lambda}{p^{\prime}}} \Delta(x)^{\frac{\lambda}{p}} d x \leq\left(\int_{\mathbb{R}} \Delta(x) d x\right)^{\frac{\lambda}{p^{\prime}}}\left(\int_{\mathbb{R}} \Delta(x)^{\frac{\lambda}{p} \frac{1}{1-\lambda / p^{\prime}}} d x\right)^{1-\frac{\lambda}{p^{\prime}}} \\
& \leq C|v|_{\mathrm{BV}}^{\frac{\lambda}{p^{\prime}}}|h|^{\frac{\lambda}{p^{\prime}}} \omega_{r}(v,|h|)_{\mu}^{\mu\left(1-\frac{\lambda}{p^{\prime}}\right)}
\end{aligned}
$$

since $\mu=\frac{\lambda}{p} \frac{1}{1-\lambda / p^{\prime}}$.

By taking a supremum for $|h| \leq s$, we can replace the left side by $\omega_{r}(v, s)_{\lambda}^{\lambda}$ and raise both sides to the power $1 / \lambda$ to obtain

$$
\omega_{r}(v, s)_{\lambda} \leq C|v|_{\mathrm{BV}}^{\frac{1}{p^{\prime}}} s \frac{1}{p^{\prime}} \omega_{r}(v, s)_{\mu}^{\frac{1}{p}},
$$

where we have used the fact that $\left(\frac{1}{\lambda}-\frac{1}{p^{\prime}}\right) \mu=\frac{1}{p}$. Therefore

$$
\begin{aligned}
\int_{0}^{\infty}\left[s^{-\beta} \omega_{r}(v, s)_{\lambda}\right]^{\sigma} \frac{d s}{s} & \leq C|v|_{\mathrm{BV}}^{\frac{\sigma}{p^{\prime}}} \int_{0}^{\infty} s^{-\beta \sigma+\frac{\sigma}{p^{\prime}}} \omega_{r}(v, s)_{\mu}^{\frac{\sigma}{p}} \frac{d s}{s} \\
& =C|v|_{\mathrm{BV}}^{\frac{\sigma}{p^{\prime}}} \int_{0}^{\infty}\left[s^{-\alpha} \omega_{r}(v, s)_{\mu}\right]^{\mu} \frac{d s}{s}
\end{aligned}
$$

since $\mu=\tau(\alpha, 1)=\sigma / p$ and $\alpha \mu=\beta \sigma-\sigma / p^{\prime}$. Raising both sides of this inequality to the power $1 / \sigma$ shows that

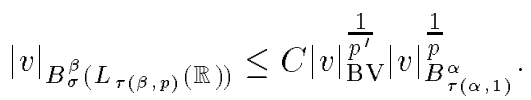

In the next theorem, we apply Lemma 5.1 to solutions of (1.1).

THEOREM 5.2. Let the flux $f$ be strictly convex and in $C^{r+1}$. If $u_{0}$ is a function of compact support in $B_{\tau(\alpha, p)}^{\alpha} \cap \mathrm{BV}(\mathbb{R})$ for some $\alpha>1$ and $1<p \leq \infty$, then for any later time $t$ the solution $u(\cdot, t)$ to $(1.1)$ is in every Besov space $B_{\tau(\beta, q)}^{\beta} \cap \mathrm{BV}(\mathbb{R})$ for all $1 \leq q<\infty$ and $0<\beta<1+(\alpha-1) / q$.

Proof. It may help the reader to refer to Figure 3 during the course of this proof. We first assume that $1<q<\infty$ and $1 \leq \beta<1+(\alpha-1) / q$, and we choose auxiliary parameters $\alpha^{\prime}$, $\beta^{\prime}$, and $s$ that satisfy $1<\alpha^{\prime}<\alpha, \beta<\beta^{\prime}<1+\left(\alpha^{\prime}-1\right) / q, \tau\left(\alpha^{\prime}, 1\right)<$ $\tau(\alpha, p)$ and

$$
\frac{1}{\tau(\beta, q)}=\beta^{\prime}+\frac{1}{s}
$$




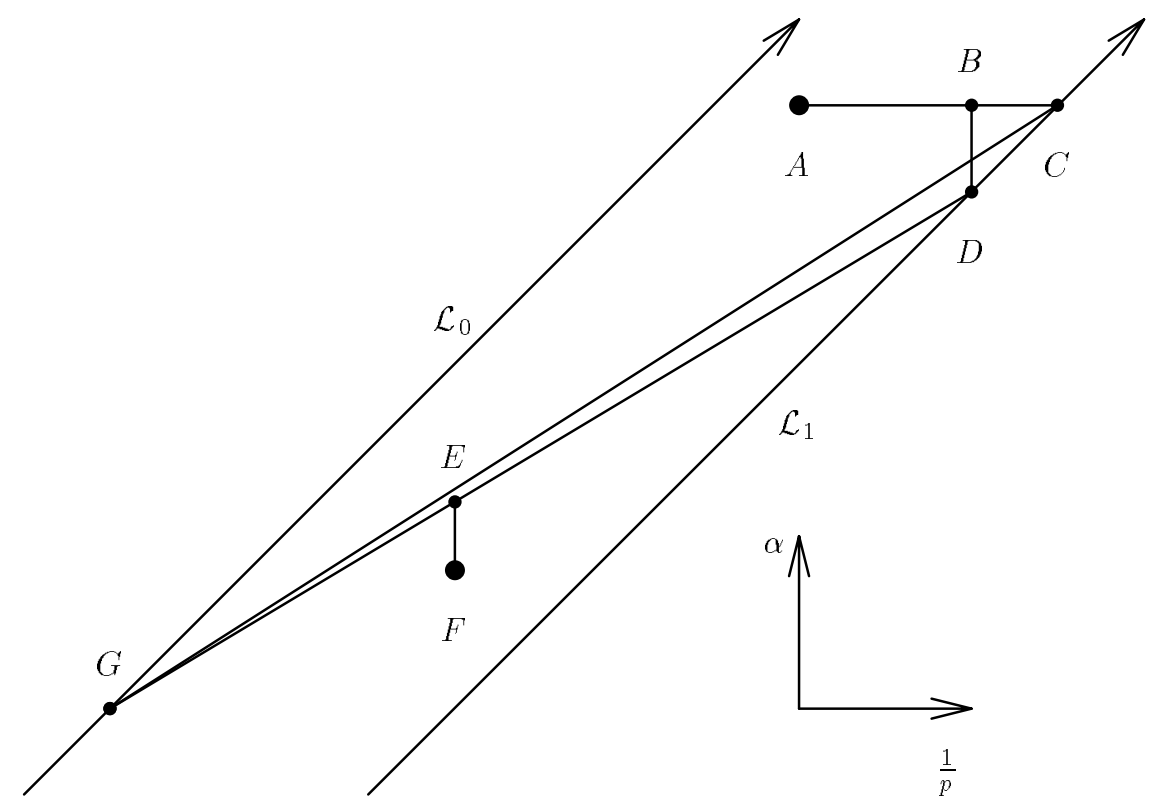

Fig. 3. The parameters and spaces of Theorem 5.2. The points and the spaces are $A: \quad\left(\frac{1}{\tau(\alpha, p)}, \alpha\right), B_{\tau(\alpha, p)}^{\alpha}\left(L_{\tau(\alpha, p)}(\mathbb{R})\right) ; B:\left(\frac{1}{\tau\left(\alpha^{\prime}, 1\right)}, \alpha\right), B_{\tau(\alpha, p)}^{\alpha}\left(L_{\tau\left(\alpha^{\prime}, 1\right)}(\mathbb{R})\right) ; C: \quad\left(\frac{1}{\tau(\alpha, 1)}, \alpha\right) ; D:$ $\left(\frac{1}{\tau\left(\alpha^{\prime}, 1\right)}, \alpha^{\prime}\right), \quad B_{\tau\left(\alpha^{\prime}, 1\right)}^{\alpha^{\prime}}\left(L_{\tau\left(\alpha^{\prime}, 1\right)}(\mathbb{R})\right) ; \quad E: \quad\left(\frac{1}{\tau\left(\beta^{\prime}, r\right)}, \beta^{\prime}\right)=\left(\frac{1}{\tau(\beta, q)}, \beta^{\prime}\right), \quad B_{r \tau\left(\alpha^{\prime}, 1\right)}^{\beta^{\prime}}\left(L_{\tau\left(\beta^{\prime}, r\right)}(\mathbb{R})\right) ; F:$ $\left(\frac{1}{\tau(\beta, q)}, \beta\right), B_{\tau(\beta, q)}^{\beta}\left(L_{\tau(\beta, q)}(\mathbb{R})\right)$; and $\left.G:(1,1), \mathrm{BV}(\mathbb{R})\right)$. The lines $\mathcal{L}_{0}$ and $\mathcal{L}_{1}$ are as in Figure 1 .

i.e., $\tau(\beta, q)=\tau\left(\beta^{\prime}, s\right)$. Note that it is always possible to choose these parameters by first choosing $\alpha^{\prime}$ close enough to $\alpha$. It follows that $1<s<\infty$.

We note that because $u_{0}$ has compact support, $u_{0} \in B_{\tau(\alpha, p)}^{\alpha}\left(L_{\tau(\alpha, p)}(\mathbb{R})\right.$ ) (Point $A$ ) implies that $u_{0} \in B_{\tau(\alpha, p)}^{\alpha}\left(L_{\tau\left(\alpha^{\prime}, 1\right)}(\mathbb{R})\right)$ (Point $\left.B\right)$, since $\tau\left(\alpha^{\prime}, 1\right)<\tau(\alpha, p)$. Now, by the embedding theorems mentioned in $\S 3, u_{0}$ is in $B_{\tau\left(\alpha^{\prime}, 1\right)}^{\alpha^{\prime}}\left(L_{\tau\left(\alpha^{\prime}, 1\right)}(\mathbb{R})\right)$ (Point D), since $\alpha^{\prime}<\alpha$.

Inequalities (5.1) and (5.2) and the fact that $u_{0} \in B_{\tau\left(\alpha^{\prime}, 1\right)}^{\alpha^{\prime}}\left(L_{\tau\left(\alpha^{\prime}, 1\right)}(\mathbb{R})\right) \cap \mathrm{BV}(\mathbb{R})$ imply that $u(\cdot, t)$ is in the same space for all $t>0$. We can apply Lemma 5.1 to see that $u(\cdot, t)$ is in $B_{s \tau\left(\alpha^{\prime}, 1\right)}^{\beta^{\prime}}\left(L_{\tau\left(\beta^{\prime}, s\right)}(\mathbb{R})\right)=B_{s \tau\left(\alpha^{\prime}, 1\right)}^{\beta^{\prime}}\left(L_{\tau(\beta, q)}(\mathbb{R})\right)$ (Point $E$ ). Finally, because $\beta<\beta^{\prime}$, a standard embedding theorem implies that $u(\cdot, t)$ is in $B_{\tau(\beta, q)}^{\beta}\left(L_{\tau(\beta, q)}(\mathbb{R})\right)$ (Point $F$ ), as required.

When $q=1$ or $\beta<1$, the theorem follows from what we have already shown and the fact that $B_{\tau(\tilde{\beta}, q)}^{\tilde{\beta}}$ is embedded in $B_{\tau(\beta, q)}^{\beta}$ for any $\beta<\tilde{\beta}$.

6. Regularity in Besov spaces. Part II: Limits on regularity. Recall that a smoothness space $X$ is a regularity space for (1.1) if $u_{0} \in X \Longrightarrow u(\cdot, t) \in X$ for all $t>0$. We have remarked that the spaces $\mathrm{BV}(\mathbb{R}) \cap B_{\tau(\alpha, 1)}^{\alpha}$ are regularity spaces for (1.1), and we gave simple arguments in the introduction to show that spaces on or above the line $\mathcal{L}_{0}$ with $\alpha>1$ or below the line $\mathcal{L}_{1}$ cannot be regularity spaces for $X$. We went on to show that if $u_{0}$ is in any space $X=B_{\tau(\alpha, p)}^{\alpha} \cap \mathrm{BV}(\mathbb{R})$ for $\alpha>1$ and $1<p<\infty$ (i.e., between the lines $\mathcal{L}_{0}$ and $\mathcal{L}_{1}$ ), then the solutions $u(\cdot, t)$ remained in $B_{\tau(\beta, q)}^{\beta}$ for $1 \leq q<\infty$ and $\beta<1+(\alpha-1) / q$ for all time; we now show that the solution 
of (1.1) is, in general, not in any space $B_{\tau(\beta, q)}^{\beta}$ with $1 \leq q<\infty$ and $\beta>1+(\alpha-1) / q$. In particular, no Besov space $B_{\sigma}^{\alpha}$ for $\alpha>1$ and any $\sigma \neq \tau(\alpha, 1)$ is a regularity space for the one-dimensional conservation law (1.1). In this section, we analyze the inviscid Burgers equation, and in the next section we treat general strictly convex fluxes $f$. In $\S 8$, we generalize these results to multivariate problems.

So we consider in this section only $f(u)=u^{2} / 2, \alpha>1$, and $\tau=\tau(\alpha, p):=$ $(\alpha+1 / p)^{-1}$ for $1<p<\infty$. For any $\alpha$ and $p$ we construct initial data $u_{0} \in \mathrm{BV} \cap B_{\tau(\alpha, p)}^{\alpha}$ such that the solution $u(x, 1)$ of $(1.1)$ at time 1 is not in any space $B_{\tau(\beta, q)}^{\beta}$ for $1 \leq q<\infty$ and $\beta>1+(\alpha-1) / q$.

It is perhaps easier to first describe the solution $u(x, 1)$ that we want to achieve at time 1 and then explain how to find suitable initial data $u_{0}$ that yields it. We construct a set of functions $\phi_{k}, k=1,2, \ldots$, each of compact support on intervals $I_{k}$ such that $\sum_{k}\left|I_{k}\right|$ is finite; our solution will be $u(\cdot, 1)=u_{1}:=\sum_{k} \phi_{k}\left(\cdot-x_{k}\right)$, where the increasing sequence of points $x_{k}$ is chosen such that $u(x, 1)$ has bounded support and the supports of $\phi_{k}\left(\cdot-x_{k}\right)$ and $\phi_{j}\left(\cdot-x_{j}\right)$ don't overlap if $j \neq k$. The graph of $\phi_{k}\left(x-x_{k}\right)$ is given in Figure 4. The right-most portion of its graph has $N_{k}$ steps with height $H_{k}:=2^{-k}$ and width $W_{k}:=2^{-\alpha k}$; on the left, a linear piece with slope $\frac{1}{2}$ connects the top of the steps with the $x$ axis. Precisely,

$$
\phi_{k}(x):= \begin{cases}0, & x \leq-2 N_{k} H_{k}, \\ \frac{1}{2} x+N_{k} H_{k}, & -2 N_{k} H_{k} \leq x \leq 0, \\ \left(N_{k}-n\right) H_{k}, & n W_{k} \leq x<(n+1) W_{k}, 0 \leq n<N_{k}, \\ 0, & N_{k} W_{k} \leq x .\end{cases}
$$

We choose $N_{k}$ as the greatest integer such that $N_{k} H_{k} \leq k^{-r}$. If $N_{k}$ is zero then $\phi_{k}$ is defined to be zero. If $N_{k}>0$ then

$$
\frac{1}{2} k^{-r} \leq N_{k} H_{k} \leq k^{-r}
$$

The integer $r$ will be given later. We have

$$
\left|I_{k}\right|=2 N_{k} H_{k}+N_{k} W_{k} \leq 3 N_{k} H_{k} \leq 3 k^{-r},
$$

so $\sum_{k}\left|I_{k}\right|<\infty$ as claimed, and a set of points $\left\{x_{k}\right\}_{k=1}^{\infty}$ can easily be chosen with the required properties.

We obtain $u_{0}(x)$ by solving the associated backward problem

$$
\begin{array}{ll}
v_{t}-\left(\frac{1}{2} v^{2}\right)_{x}=0, & x \in \mathbb{R}^{d}, \quad t>0, \\
v(x, 0)=v_{0}(x):=u(x, 1), & x \in \mathbb{R}^{d} .
\end{array}
$$

Then we take $u_{0}:=v(\cdot, 1)$. Each jump in $v_{0}$ smooths into a linear rarefaction wave with slope -1 , and the linear piece on the left of each $\phi_{k}$ evolves into a steeper profile with slope 1 , but not yet a shock, at time 1 . Thus, $u_{0}(x):=v(x, 1)=\sum_{k} \psi_{k}\left(x-x_{k}\right)$, where the continuous function $\psi_{k}$ takes the values

$$
\begin{cases}0, & x=-2 N_{k} H_{k}, \\ \left(N_{k}-n\right) H_{k}, & x=n W_{k}-\left(N_{k}-n\right) H_{k} \text { and } \\ & x=(n+1) W_{k}-\left(N_{k}-n\right) H_{k}, \quad 0 \leq n<N_{k}, \\ 0, & x=N_{k} W_{k},\end{cases}
$$



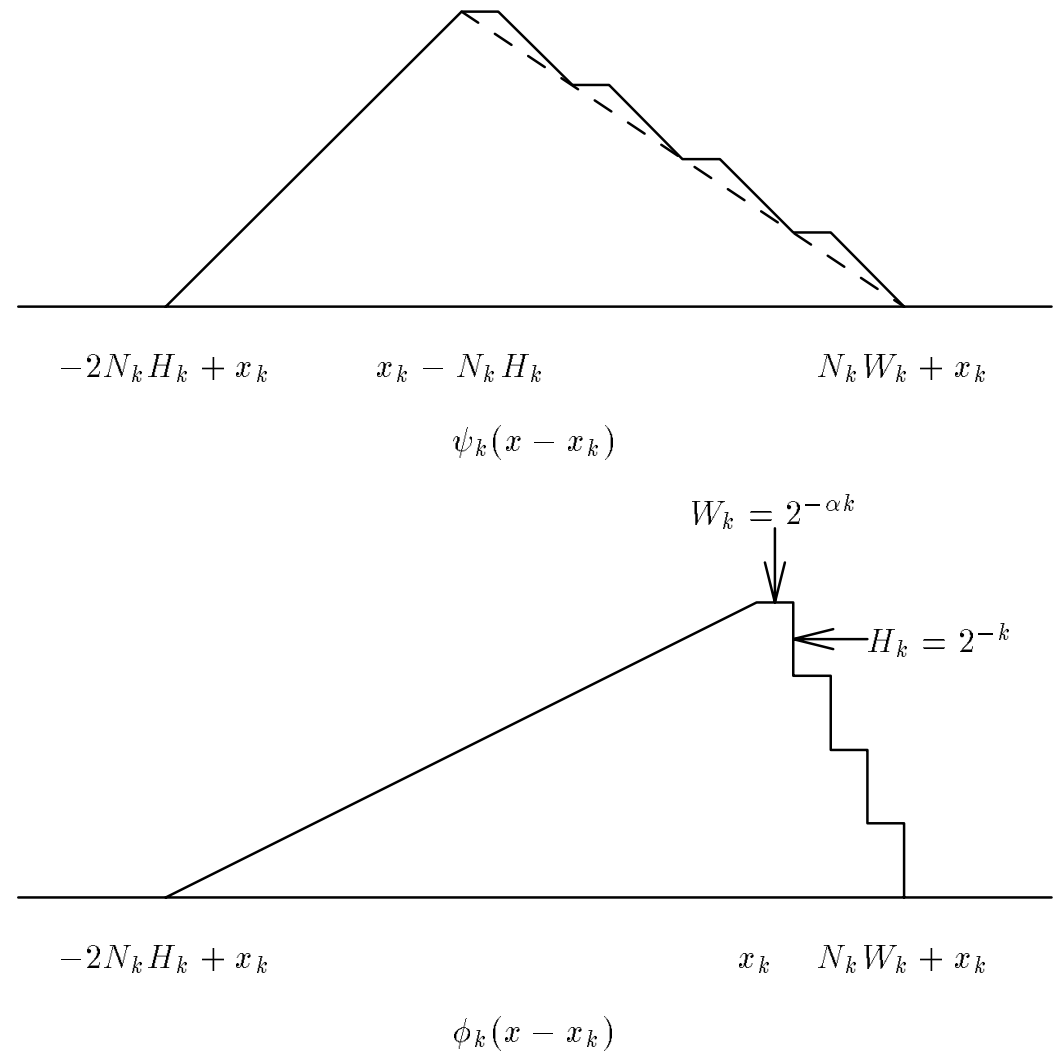

Fig. 4. The functions $\phi_{k}\left(x-x_{k}\right)$ and $\psi_{k}\left(x-x_{k}\right)$, from which $u_{0}(x)=\sum_{k} \psi_{k}\left(x-x_{k}\right)$ and $u(x, 1)=\sum_{k} \phi_{k}\left(x-x_{k}\right)$. The dashed line indicates the linear approximation to $\psi_{k}\left(x-x_{k}\right)$ in Theorem 6.1.

is linear between these values, and is zero outside the interval $\left[-2 N_{k} H_{k}, N_{k} W_{k}\right]$. Thus, on the right, $\psi_{k}$ consists of linear pieces with slopes alternating between 0 and -1 . See Figure 4. Note that $\psi_{k}$ has $2 N_{k}+1$ linear pieces and $\phi_{k}$ has $N_{k}+1$ linear pieces.

It is easy to justify that $u_{1}$ is the solution to (1.1) when $t=1$ with initial data $u_{0}$. For example, in the description of the solution given by Lax (see our $\S 2$ ), for $t<1$ there is a unique solution $y(x, t)$ to $(2.3)$. When $t=1$ there is a unique solution to (2.3) except at breakpoints of $u_{1}$. These correspond to the jumps in $u_{1}$.

We fix $\alpha>1$ and $1<p<\infty$ and let $r$ be the smallest integer that satisfies $(r-1) \tau(\alpha, p) / p>1$, or, equivalently, $r>\alpha p+2$.

THEOREM 6.1. Let $1<p<\infty$ and $\alpha>1$ be fixed. The function $u_{0}$ defined above is in $B_{\tau(\alpha, p)}^{\alpha}$, for $\tau(\alpha, p):=(\alpha+1 / p)^{-1}$ while the function $u_{1}=u(\cdot, 1)$ with $u$ the solution to (1.1) for this $u_{0}$ and $f(u):=u^{2} / 2$ is not in any space $B_{\tau(\beta, q)}^{\beta}$ for any $1<q<\infty$ and any $\beta>1+(\alpha-1) / q$.

Proof. (i) We first show that $u_{0} \in B_{\tau(\alpha, p)}^{\alpha}$. For this purpose, we use the seminorm (3.5) for $B_{\tau(\alpha, p)}^{\alpha}$ that is defined using the approximation errors $\sigma_{2^{j}}\left(u_{0}\right)_{p}$. We fix a value of $n \geq 1$ such that $2^{n} \geq n^{r}$ and we bound $\sigma_{m}\left(u_{0}\right)$ for $m:=2^{n+3}$ by constructing a piecewise linear approximant $S$ to $u_{0}$ as follows.

Recall that

$$
\operatorname{supp}\left(\phi_{k}\right)=\operatorname{supp}\left(\psi_{k}\right)=I_{k}=\left[-2 N_{k} H_{k}, N_{k} W_{k}\right]
$$


Outside of $\bigcup_{k}\left(x_{k}+I_{k}\right)$, we define $S$ to be zero. Let $z_{k}:=x_{k}+N_{k} W_{k}$ be the right endpoint of the support interval $x_{k}+I_{k}$ of $\psi_{k}\left(x-x_{k}\right)$. On each $x_{k}+I_{k}$ for $1 \leq k<n$ (i.e., where $u_{0}$ is largest), we define $S(x):=u_{0}(x)=\psi_{k}\left(x-x_{k}\right)$. Then $S$ has at most $\sum_{j=1}^{n-1}\left(2 N_{j}+2\right) \leq 2^{n+1}+2 n \leq 2^{n+2}$ linear pieces to the left of the point $z_{n-1}$. To the right of $z_{n-1}$ we define $S$ as follows. On any interval $x_{k}+I_{k}$, with $n \leq k \leq 2^{n}$ (where $u_{0}$ is of moderate size), we define $S$ to be the continuous, piecewise linear function that passes through the points $\left(x_{k}-2 N_{k} H_{k}, 0\right),\left(x_{k}-N_{k} H_{k}, N_{k} H_{k}\right)$, and $\left(x_{k}+N_{k} W_{k}, 0\right)$ (the dashed line in Figure 4). To the right of $z_{2^{n}}$ (where $u_{0}$ is smallest), we define $S$ to be identically zero; we call this semi-infinite interval $I_{\infty}$. Then $S$ has at most $3 \cdot 2^{n}$ breakpoints to the right of $z_{n-1}$ and hence at most $m=2^{n+3}$ breakpoints in all.

We consider next the error $E(x):=\left|u_{0}(x)-S(x)\right|$ at points where $E$ is not identically zero. On any interval $x_{k}+I_{k}, k=n, \ldots, 2^{n}$, the error is no greater than $W_{k}$ (since the slope of the dashed line in Figure 4 is greater than -1 ) and $E$ is nonzero on a set of measure at most $N_{k}\left(W_{k}+H_{k}\right) \leq 2 N_{k} H_{k} \leq 2 k^{-r}$. Hence

$$
\int_{x_{k}+I_{k}} E(x)^{p} d x \leq 2 W_{k}^{p} k^{-r} \leq 2 \cdot 2^{-k \alpha p} k^{-r} .
$$

On the other hand, on $I_{\infty}, E(x) \leq N_{2^{n}} H_{2^{n}} \leq 2^{-n r}$ and $E$ is nonzero on a set of measure not exceeding

$$
\sum_{k=2^{n}}^{\infty}\left|I_{k}\right| \leq 3 \sum_{k=2^{n}}^{\infty} \frac{1}{k^{r}} \leq C 2^{-n(r-1)},
$$

(see (6.2)) with $C$ (here and later in this proof) depending only on $r$, since $r>1$. This gives

$$
\int_{I_{\infty}} E(x)^{p} d x \leq C 2^{-n r p} 2^{-n(r-1)}
$$

Adding the estimates (6.3) and (6.4), we obtain for $m=2^{n+3}$ and $n$ sufficiently large,

$$
\begin{aligned}
\sigma_{m}\left(u_{0}\right)_{p}^{p} \leq\left\|u_{0}-S\right\|_{p}^{p} & \leq C 2^{-n r p} 2^{-n(r-1)}+2 \sum_{k=n}^{2^{n}} k^{-r} 2^{-k \alpha p} \\
& \leq 2^{-n r p} 2^{-n(r-1)}+C 2^{-n \alpha p} n^{-r} \\
& \leq C 2^{-n \alpha p} n^{-r}
\end{aligned}
$$

since $r \geq \alpha$ and $r>1$. This inequality also holds (trivially) for all $n$ by simply adjusting the constant $C$. Using this and the monotonicity of $\sigma_{j}\left(u_{0}\right)_{p}$, we obtain that

$$
\sum_{n=1}^{\infty}\left[2^{n \alpha} \sigma_{2^{n}}\left(u_{0}\right)_{p}\right]^{\tau(\alpha, p)} \leq C \sum_{n=1}^{\infty} n^{-r \tau(\alpha, p) / p}<\infty
$$

since $r \tau(\alpha, p) / p>1$ by our definition of $r$. This shows that $u_{0}$ is in $B_{\tau(\alpha, p)}^{\alpha}$ and completes the proof of (i).

(ii) We show next that $u_{1}=u(\cdot, 1) \notin B_{\tau(\beta, q)}^{\beta}$ for any $1 \leq q<\infty$ and $\beta>$ $1+(\alpha-1) / q$ by giving a lower bound on $\omega_{r^{\prime}}\left(u_{1}, t\right)_{\tau(\beta, q)}$ for any fixed $r^{\prime}>\beta$. We consider any $k$ for which $\phi_{k}$ is not identically zero, and we examine $\Delta_{h}^{r^{\prime}}\left(\phi_{k}, x\right)$ for $h:=h_{k}:=W_{k} / r^{\prime}$ and $\nu W_{k}-h<x<\nu W_{k}, \nu=1, \ldots, N_{k}$, i.e., just to the left of each 
jump of height $H_{k}$ in $\phi_{k}$. Since $\Delta_{h}^{r^{\prime}}(g, x)=0$ for any constant function $g$, we have for $\nu W_{k}-h<x<\nu W_{k}$,

$$
\Delta_{h}^{r^{\prime}}\left(\phi_{k}, x\right)=\Delta_{h}^{r^{\prime}}\left(\phi_{k}-\left(N_{k}-\nu\right) H_{k}, x\right)=(-1)^{r^{\prime}} H_{k}=(-1)^{r^{\prime}} 2^{-k}
$$

since all values of $\phi_{k}(x+j h)-\left(N_{k}-\nu\right) H_{k}=0$ in (3.1) except for $j=0$. This holds for $x$ on a set of measure $N_{k} h=N_{k} W_{k} / r^{\prime} \geq C 2^{k} k^{-r} 2^{-\alpha k}=C k^{-r} 2^{-(\alpha-1) k}$.

From (6.5), we derive for $\tau:=\tau(\beta, q):=(\beta+1 / q)^{-1}$,

$$
\omega_{r^{\prime}}\left(u_{1}, h_{k}\right)_{\tau}^{\tau} \geq\left|H_{k}\right|^{\tau} k^{-r} 2^{-(\alpha-1) k}=C 2^{k(1-\tau-\alpha)} k^{-r},
$$

for all $k$ sufficiently large, say $k \geq k_{0}$. Using the monotonicity of $\omega_{r^{\prime}}\left(u_{1}, t\right)_{\tau}$ in $t$, dividing the interval of integration in (3.2) into intervals $\left[h_{k+1}, h_{k}\right)$ and discretizing the integral yields

$$
\begin{aligned}
\left|u_{1}\right|_{B_{\tau}^{\beta}}^{\tau} & \geq C \sum_{k=k_{0}}^{\infty} h_{k}^{-\beta \tau} \omega_{r^{\prime}}\left(u_{1}, h_{k}\right)_{\tau}^{\tau} \\
& \geq C \sum_{k=k_{0}}^{\infty} k^{-r} 2^{k(\beta \tau \alpha+1-\tau-\alpha)}=\infty .
\end{aligned}
$$

because

$$
\beta \tau \alpha+1-\tau-\alpha=\beta \frac{q}{\beta q+1} \alpha+1-\frac{q}{\beta q+1}-\alpha=\frac{\beta q+1-q-\alpha}{\beta q+1}>0 .
$$

Hence $u_{1} \notin B_{\tau(\beta, q)}^{\beta}$.

The previous theorem can be used together with embedding theorems for Besov spaces to show that none of the Besov spaces $B_{s}^{\alpha}\left(L_{\tau}\right)$ with $(\alpha+1)^{-1}<\tau<\alpha^{-1}$ are regularity spaces. That is, we can allow any value of $s$. A modification of the construction of the theorem allows this conclusion for $\tau=\alpha^{-1}$. We already remarked that no such space with $\tau>1 / \alpha$ or $\tau<1 /(\alpha+1)$ is a regularity space. We leave these details to the reader.

7. More general fluxes. We shall next show that the results of the previous section are valid for more general fluxes $f$. We shall assume in this section that $f$ is a strictly convex function on $\mathbb{R}$. Then $a(u):=f^{\prime}(u)$ is strictly increasing and has an inverse $b:=a^{-1}$ under composition of functions. We shall assume that $a(0)=0$ and therefore $b(0)=0$ (this assumption could be removed with a suitable change in the construction below).

If $\alpha>1$, let $r, u_{0}$, and $u_{1}$ be defined as in the previous section. We consider the solution $v(x, t)$ to (1.1) for the flux $f$ and the initial condition

$$
v_{0}(x):=b\left(u_{0}(x)\right), \quad x \in \mathbb{R} .
$$

The same argument we have given in $\S 6$ can be applied here to show that the solution $v(x, t)$ of $(1.1)$ for $t \leq 1$ with data $v_{0}$ is the same as $b(u(x, t))$, where $u(x, t)$ is defined in the previous section.

The next two theorems will show that for every $1<p<\infty$ and $\alpha>1$, the function $v_{0}$ is in $B_{\tau(\alpha, p)}^{\alpha}$, but $v(\cdot, 1)=v_{1}$ is not in any space $B_{\tau(\beta, q)}^{\beta}$ for any $1<q<\infty$ and $\beta>1+(\alpha-1) / q$. We shall assume in these theorems that the flux $f$ is in $C^{r+1}$; it follows that $a$ and $b$ are in $C^{r}$. 
THEOREM 7.1. Let $\alpha, r, u_{0}$, and $\tau:=\tau(\alpha, p)$ be defined as above, and let $1<$ $p<\infty$. If $b$ is in $C^{r}[0,1]$, then $v_{0}$ is in $B_{\tau(\alpha, p)}^{\alpha}$.

Proof. This proof is similar to Part (i) of Theorem 6.1.

It is reasonable to expect that $v_{0}$ will be as smooth as $u_{0}$ since $b$ is smooth but the proof is not completely trivial. We shall estimate the error in approximating $v_{0}$ by piecewise polynomials of order $r$ with $m$ pieces. That is, we shall estimate the error $\sigma_{m}\left(v_{0}\right)_{p}$ in approximating $v_{0}$ by the elements of $\Sigma_{m, r}$ in the $L_{p}(\mathbb{R})$ norm. We first note that since $b(0)=0$ and $0 \leq u_{0}(x) \leq 1$, for all $x$, we have $0 \leq v_{0}(x) \leq\left\|b^{\prime}\right\|_{L_{\infty}[0,1]} u_{0}(x)$ for all $x \in \mathbb{R}$. This implies that $v_{0} \in L_{p}(\mathbb{R})$.

We fix an integer $n \geq 1$ and we estimate $\sigma_{m}\left(v_{0}\right)_{p}$ for $m \geq C_{0} 2^{n}$ with $C_{0}$ an absolute constant that is specified in the course of the proof. We let $\Psi_{1}:=\sum_{k=1}^{n-1} \psi_{k}(\cdot-$ $\left.x_{k}\right), \Psi_{2}:=\sum_{k=n}^{2^{n}} \psi_{k}\left(\cdot-x_{k}\right)$, and $\Psi_{3}:=\sum_{k>2^{n}} \psi_{k}\left(\cdot-x_{k}\right)$. These functions have disjoint supports. From the definition of $v_{0}$, we have

$$
v_{0}=b\left(\Psi_{1}\right)+b\left(\Psi_{2}\right)+b\left(\Psi_{3}\right)=: b_{1}+b_{2}+b_{3} .
$$

We first estimate $\sigma_{m}\left(b_{1}\right)_{p}$. Recall that $\Psi_{1}$ is a piecewise linear function on $\mathbb{R}$ with no more than $C 2^{n}$ breakpoints. We shall now show that one can add at most an additional $C_{0} 2^{n}$ breakpoints so that for any interval $I$ in the resulting partition, $\Psi_{1}(I)$ is contained in an interval of length $2^{-n}$. Indeed, the variation of $\psi_{k} \leq 2 / k^{r}$, for $k=1, \ldots, n$; hence, we need only insert at most $2 k^{-r} 2^{n}+2$ new breakpoints for each $k=1, \ldots, n-1$ to obtain the desired partition. For each of these intervals $I$, we let $P_{I}$ be the Taylor polynomial to $b$ of order $r$ expanded at the center of $\Psi_{1}(I)$. Then $P_{I}\left(\Psi_{1}\right)$ is a polynomial of order $r$ on $I$. We define the piecewise polynomial function $S_{1}$ by $S_{1}:=P_{I}\left(\Psi_{1}\right)$ for each $I$. Then,

$$
\left\|b_{1}-S_{1}\right\|_{L_{\infty}(I)}=\left\|b\left(\Psi_{1}\right)-P_{I}\left(\Psi_{1}\right)\right\|_{L_{\infty}(I)} \leq\left\|b^{(r)}\right\|_{L_{\infty}[0,1]} 2^{-n r}
$$

Since $b_{1}$ and $S_{1}$ have compact support, it follows that

$$
\sigma_{m}\left(b_{1}\right)_{p} \leq C 2^{-n r}, \quad m \geq C_{0} 2^{n}
$$

with $C$ not depending on $n$.

We can estimate $\sigma_{m}\left(b_{2}\right)$ in a similar way. We have shown in the proof of Theorem 6.1 that there is a piecewise linear function $\tilde{\Psi}_{2}$ with at most $3 \cdot 2^{n}$ pieces that satisfies

$$
\left\|\Psi_{2}-\tilde{\Psi}_{2}\right\|_{L_{\infty}(\mathbb{R})} \leq 2^{-n \alpha} .
$$

Moreover, $\tilde{\Psi}_{2}=\sum_{k=n}^{2^{n}} \tilde{\psi}_{k}\left(\cdot-x_{k}\right)$ with each $\tilde{\psi}_{k}\left(\cdot-x_{k}\right)$ a piecewise linear function with 4 pieces and $\operatorname{Var}\left(\tilde{\psi}_{k}\right)=\operatorname{Var}\left(\psi_{k}\right) \leq 2 k^{-r}$. Therefore, as in the previous case of $\Psi_{1}$, we can add new breakpoints and obtain a partition of $\mathbb{R}$ into at most $C_{0} 2^{n}$ intervals $I$ such that $\tilde{\Psi}_{2}$ is linear on $I$ and $\tilde{\Psi}_{2}(I)$ is contained in an interval of length $\leq 2^{-n}$. If $P_{I}$ denotes the Taylor polynomial of order $r$ of $b$ expanded about the center of $\tilde{\Psi}_{2}(I)$, then $P_{I}\left(\tilde{\Psi}_{2}\right)$ is a polynomial of order $r$ on $I$. The piecewise polynomial function $S_{2}$ is defined to be $P_{I}\left(\tilde{\Psi}_{2}\right)$ on each $I$. Then, for each of the intervals $I$, we have

$$
\begin{aligned}
\left\|b_{2}-S_{2}\right\|_{L_{\infty}(I)} & \leq\left\|b\left(\Psi_{2}\right)-b\left(\tilde{\Psi}_{2}\right)\right\|_{L_{\infty}(I)}+\left\|b\left(\tilde{\Psi}_{2}\right)-P_{I}\left(\tilde{\Psi}_{2}\right)\right\|_{L_{\infty}(I)} \\
& \leq\left\|b^{\prime}\right\|_{L_{\infty}[0,1]} 2^{-n \alpha}+\left\|b^{(r)}\right\|_{L_{\infty}[0,1]} 2^{-n r} \leq C 2^{-n \alpha}
\end{aligned}
$$

with $C$ independent of $n$. Now, by (6.2), $b_{2}$ and $S_{2}$ vanish outside of a set of measure at most $C n^{-r+1}$ with $C$ depending only on $r$. Hence, from (7.3),

$$
\left\|b_{2}-S_{2}\right\|_{L_{p}(\mathbb{R})} \leq C n^{(-r+1) / p} 2^{-n \alpha} .
$$


It follows that

$$
\sigma_{m}\left(b_{2}\right)_{p} \leq C n^{(-r+1) / p} 2^{-n \alpha}, \quad m \geq C_{0} 2^{n} .
$$

Since $b(0)=0$ and $\left\|\Psi_{3}\right\|_{L_{\infty}(\mathbb{R})}=\sup _{k>2^{n}}\left\|\psi_{k}\right\|_{L_{\infty}(\mathbb{R})} \leq 2^{-n r}$, we have

$$
\left\|b_{3}\right\|_{L_{\infty}(\mathbb{R})} \leq\left\|b^{\prime}\right\|_{L_{\infty}[0,1]}\left\|\Psi_{3}\right\|_{L_{\infty}(\mathbb{R})} \leq\left\|b^{\prime}\right\|_{L_{\infty}[0,1]} 2^{-n r} .
$$

Because $b_{3}$ has compact support, we have

$$
\sigma_{m}\left(b_{3}\right)_{p} \leq C 2^{-n r}, \quad m \geq 1 .
$$

Now, $v_{0}=b_{1}+b_{2}+b_{3}$, and therefore the estimates (7.2), (7.4), and (7.5) give

$$
\sigma_{m}\left(v_{0}\right)_{p} \leq C n^{(-r+1) / p} 2^{-n \alpha}, \quad m \geq C_{0} 2^{n} .
$$

From our assumption on $r$, we have $\tau(r-1) / p>1$ and therefore

$$
\sum_{n=1}^{\infty}\left[2^{n \alpha} \sigma_{2^{n}}\left(v_{0}\right)_{p}\right]^{\tau}<\infty
$$

From the characterization (3.5), we obtain that $v_{0} \in B_{\tau(\alpha, p)}^{\alpha}$.

We shall next show that $v_{1}:=v(\cdot, 1)$ is not in any $B_{\tau(\beta, q)}^{\beta}$, for $1 \leq q<\infty$ and $\beta>(\alpha-1) / q+1$. For this, we shall assume that

$$
b^{\prime}(x) \geq c, \quad x \in(0,1)
$$

for some $c>0$.

THEOREM 7.2. Under the assumptions of Theorem 7.1 and the added assumption (7.6), we have $v_{1} \notin B_{\tau(\beta, q)}^{\beta}$ for all $1 \leq q<\infty$ and $\beta>(\alpha-1) / q+1$.

Proof. The widths of the constant states in $b\left(\phi_{k}\right)$ are the same as for $\phi_{k}$, and because of assumption (7.6), the heights of the jumps are $\geq c 2^{-k}$. Therefore, the same argument as given in the proof of Part (ii) of Theorem 6.1 shows that (6.7) holds with $v_{1}=b\left(u_{1}\right)$ in place of $u_{1}$. Hence $v_{1}$ is not in $B_{\tau(\beta, q)}^{\beta}$.

In summary, Theorems 7.1 and 7.2 give

THEOREM 7.3. Given that $\alpha>1$ and $1<p \leq \infty$ and that the flux $f$ to the univariate conservation law (1.1) has derivative $a(u)=f^{\prime}(u)$, which is strictly increasing and whose inverse function $b$ is in $C^{r}$ with $(r-1) \tau(\alpha, p) / p>1$, and also satisfies (7.6), the initial condition $v_{0}=b\left(u_{0}\right)$ is in $B_{\tau(\alpha, p)}^{\alpha}$ but the solution $v(\cdot, 1)$ to (1.1) at time $t=1$ for this initial condition is not in any $B_{\tau(\beta, q)}^{\beta}$, for $1 \leq q<\infty$ and $\beta>(\alpha-1) / q+1$. Consequently, none of the spaces $B_{\tau}^{\alpha}\left(L_{\tau}\right), \tau \neq 1 /(\alpha+1)$ are regularity spaces for (1.1).

8. Regularity in several space dimensions. We shall next consider the regularity of the solution to the conservation law (1.1) in several space dimensions. The proof that the spaces $B_{\tau}^{\alpha}, \tau=(\alpha+1)^{-1}$, are regularity spaces for conservation laws in one space dimension rests on the fact that they arise in the characterization of approximation classes for methods of nonlinear approximation in $L_{1}(\mathbb{R})$ such as wavelets and free knot splines. The Besov spaces $B_{\tau}^{\alpha}\left(L_{\tau}\left(\mathbb{R}^{d}\right)\right), \tau=(\alpha / d+1)^{-1}$ play the analogous role in nonlinear approximation in several space dimensions. For example, they arise in the characterization of nonlinear approximation by wavelet sums (see [4]). One might expect therefore that they are regularity spaces for conservation laws in several space dimensions. We shall show that this is not the case when $\alpha>1$. 
We assume that $a(u):=f^{\prime}(u)$ is a continuously differentiable mapping from $\mathbb{R} \rightarrow \mathbb{R}^{d}$ and

$$
\begin{array}{cl}
\text { (i) } & a(0)=0 \\
\text { (ii) } & a^{\prime}(0) \neq 0
\end{array}
$$

A slight change in the argument given below would allow the point 0 to be replaced by any other point $x \in \mathbb{R}$.

We write $a(u)=:\left(a_{1}(u), \ldots, a_{d}(u)\right)$. Without loss of generality, we can assume that $a_{1}^{\prime}(0) \neq 0$ and $a_{1}^{\prime}(u)>0$, in a half- neighborhood [0, $\left.\eta\right]$ of 0 . In order to utilize our previous notation, we shall assume that $a_{1}(\eta)=1$. However, a simple modification of the arguments given below would treat the general case of $\eta$. We denote by $b_{1}$ the inverse function (under composition of functions) to $a_{1}$ on $[0, \eta]$. Then, $b_{1}$ is defined on $[0,1]$ and satisfies condition (7.6).

THEOREM 8.1. Let $\alpha>1$ and $0<\tau \leq \infty$. If $b_{1} \in C^{r}[0,1]$ for some sufficiently large integer $r>\max (\alpha, d)$ (described in Part (ii) of the proof below), then the space $B_{\tau}^{\alpha}\left(L_{\tau}\left(\mathbb{R}^{d}\right)\right)$ is not a regularity space for the conservation law (1.1).

Proof. We shall consider the following three cases.

(i) $\alpha<d(1 / \tau-1)_{+}$.

In this case, the space $B_{\tau}^{\alpha}\left(L_{\tau}\left(\mathbb{R}^{d}\right)\right)$ contains functions that are not locally integrable and hence this space cannot be a regularity space for (1.1).

(ii) $\alpha>1, \alpha \geq d(1 / \tau-1)_{+}$, and $\alpha<1 / \tau$.

We use our previous univariate notation $\tau(\alpha, p):=(\alpha+1 / p)^{-1}$. In this case, we can write $\tau=\tau(\alpha, p)$ for some $p$ with $1<p<\infty$. We shall show that there is an initial conditions $w_{0}$ of compact support that is in $B_{\tau}^{\alpha}\left(L_{\tau}\left(\mathbb{R}^{d}\right)\right)$ but the solution $w(\cdot, 1)=E(1) w_{0}$ to $(1.1)$ is not in $B_{\tau}^{\alpha}\left(L_{\tau}\left(\mathbb{R}^{d}\right)\right)$.

We shall utilize the univariate construction of $\S 7$ with some modifications. For our fixed values of $\alpha$ and $p$, we assume that $r$ is chosen as in $\S \S 6$ and 7 . Then, the construction of $\S 6$ applies and we let $u_{0}$ be the univariate function given in that section. Further, we let $v(\cdot, t)$ be the solution given in $\S 7$ to the univariate conservation law (1.1) with initial condition $v_{0}:=b_{1}\left(u_{0}\right)$ and transport velocity $a_{1}$. We recall that we have shown in $\S 7$ that $v_{0} \in B_{\tau}^{\alpha}\left(L_{\tau}(\mathbb{R})\right)$ but $v_{1}:=v(\cdot, 1)$ is not in any of the spaces $B_{\tau(\beta, q)}^{\beta}$ for $1 \leq q<\infty$ and $\beta>1+(\alpha-1) / q$. In particular $v_{1}$ is not $B_{\tau}^{\alpha}\left(L_{\tau}(\mathbb{R})\right)$.

We consider now the multivariate conservation law (1.1) with the initial condition

$$
w_{0}(x):=v_{0}\left(x_{1}\right) \varphi\left(x_{2}, \ldots, x_{d}\right), \quad x=\left(x_{1}, \ldots, x_{d}\right) \in \mathbb{R}^{d},
$$

with $\varphi\left(x_{2}, \ldots, x_{d}\right)=\phi\left(x_{2}\right) \ldots \phi\left(x_{d}\right)$ and $\phi$ a compactly supported $C^{\infty}(\mathbb{R})$ function that is one on a sufficiently large (to be chosen momentarily) interval $I$ centered at 0 and satisfying $\|\phi\|_{L_{\infty}(\mathbb{R})}=1$. We denote by $Q$ the cube $I^{d}$. We let $w=w(x, t)$ denote the solution to $(1.1)$ at time $t$ with initial condition $w_{0}$.

Let $\ell \geq 1$ be such that $v_{0}$ vanishes outside of $[-\ell, \ell]$. We claim that if the sidelength of $Q$ is chosen sufficiently large then

$$
w(x, t)=v\left(x_{1}, t\right), \quad \text { a.e. } x \in[-2 \ell, 2 \ell]^{d}, 0 \leq t \leq 1 .
$$

We now prove this claim. Since $v_{0}$ (and hence $w_{0}$ ) has compact range, for any $x \in \mathbb{R}^{d},\left|a\left(w_{0}(x)\right)\right| \leq C_{0}$ with $C_{0}$ an absolute constant and $|\cdot|$ denoting Euclidean distance. Hence, the transport velocity vector always has length bounded by $C_{0}$.

Now, given $x \in[-2 \ell, 2 \ell]^{d}$ and $0<t<1$, we consider all points $y$ that can be transported to $x$, that is, $y$ should satisfy

$$
x=y+\operatorname{ta}\left(w_{0}(y)\right) .
$$


If $y$ and $z$ are both solutions to (8.3), then when $Q$ is large enough both points $y$ and $z$ would have to come from $Q$ (because of our estimate for the size of the transport velocity). Then, $\varphi\left(y_{2}, \ldots, y_{d}\right)=1$ and similarly for $z$. Hence the first components of the vectors in (8.3) gives $y_{1}+t u_{0}\left(y_{1}\right)=x_{1}=z_{1}+t u_{0}\left(z_{1}\right)$. We have already noted in the univariate analysis of $\S 6$, that this implies $y_{1}=z_{1}$. Hence, $(8.1)$ gives $w_{0}(y)=w_{0}(z)$ and therefore (8.3) implies $y=z$. Thus, the function $\tilde{w}$ defined by

$$
\tilde{w}(x, t):=w_{0}(y)=v_{0}\left(y_{1}\right)=v\left(x_{1}, t\right)
$$

with $y$ the solution to $(8.3)$ is well defined for $x \in[-2 \ell, 2 \ell]^{d}$ and $\tilde{w}:=\tilde{w}(x, t)$ satisfies the implicit equation

$$
\tilde{w}=w_{0}(x-\operatorname{ta}(\tilde{w})), \quad x \in[-2 \ell, 2 \ell]^{d}, 0 \leq t<1 .
$$

A direct calculation shows that $\tilde{w}$ is a weak solution to $(1.1)$ on $[-2 \ell, 2 \ell]^{d} \times[0,1)$, and since $\tilde{w}$ is continuous and piecewise $C^{r}$ on subdomains of $[-2 \ell, 2 \ell]^{d} \times[0,1)$ with smooth boundaries, $\tilde{w}$ is an entropy solution of (1.1) in this region.

If we let $t \rightarrow 1$ then $w(\cdot, t)$ converges in $L_{1}\left(\mathbb{R}^{d}\right)$ to $w_{1}:=w(\cdot, 1)$. On the other hand, as $t \rightarrow 1, w(x, t)=v\left(x_{1}, t\right)$ converges to $v_{1}\left(x_{1}\right)$ a.e. on $[-2 \ell, 2 \ell]^{d}$. This shows that $w_{1}(x)=v_{1}\left(x_{1}\right)$, a.e. $x \in[-2 \ell, 2 \ell]^{d}$ and verifies our claim for $t=1$.

To complete the proof of the theorem in this case, we shall estimate the Besov norms of $w_{0}$ and $w_{1}$. We first show that $w_{1}$ is not in $B_{\tau}^{\alpha}\left(L_{\tau}\left(\mathbb{R}^{d}\right)\right)$. It is enough to consider differences $h=h_{1} e_{1}, e_{1}:=(1,0, \ldots, 0)$ in the first coordinate direction, with $0<h_{1} \leq 1 / r$. Then, $\Delta_{h}^{r}\left(w_{1}, x\right)=\Delta_{h_{1}}^{r}\left(v_{1}, x_{1}\right)$ whenever $\Delta_{h_{1}}^{r}\left(v_{1}, x_{1}\right) \neq 0$ and $x \in[-2 \ell, 2 \ell]^{d}$. Hence,

$$
\left\|\Delta_{h}^{r}\left(w_{1}, \cdot\right)\right\|_{L_{\tau}\left(\mathbb{R}^{d}\right)} \geq(4 \ell)^{\frac{d-1}{\tau}}\left\|\Delta_{h_{1}}^{r}\left(v_{1}, \cdot\right)\right\|_{L_{\tau}(\mathbb{R}), \quad 0<h_{1} \leq 1 / r .}
$$

It follows that $\omega_{r}\left(w_{1}, s\right)_{\tau} \geq \omega_{r}\left(v_{1}, s\right)_{\tau}, 0<s \leq 1 / r$. Now, we know from Theorem 6.3 that $v_{1}$ is not in the Besov space $B_{\tau}^{\alpha}\left(L_{\tau}(\mathbb{R})\right)$ and therefore, since $r>\alpha$, (by an earlier remark of $\S 3$ )

$$
\int_{0}^{1 / r}\left[s^{-\alpha} \omega_{r}\left(v_{1}, s\right)_{\tau}\right]^{\tau} \frac{d s}{s}=\infty
$$

We can replace $v_{1}$ by $w_{1}$ in (8.5) and conclude that $w_{1}$ is not in $B_{\tau}^{\alpha}\left(L_{\tau}\left(\mathbb{R}^{d}\right)\right)$.

Next, we show that $w_{0}$ is in $B_{\tau}^{\alpha}\left(L_{\tau}\left(\mathbb{R}^{d}\right)\right)$. Let $s>0$ and let $h=\left(h_{1}, \ldots, h_{d}\right) \in \mathbb{R}^{d}$ satisfy $|h| \leq s$. We define the translation operator $T(h)$ by $T(h) g:=g(\cdot+h), h \in \mathbb{R}^{d}$. We define the difference operator $D_{k}$ by $D_{k} g:=g\left(\cdot+h_{1} e_{1}+\cdots+h_{k} e_{k}\right)-g\left(\cdot+h_{1} e_{1}+\right.$ $\left.\cdots+h_{k-1} e_{k-1}\right)$ for any function $g$ on $\mathbb{R}^{d}$. Then, $\Delta_{h}=\sum_{k=1}^{d} D_{k}$. Therefore

$$
\Delta_{h}^{r}=\sum D_{k_{1}} \ldots D_{k_{r}}
$$

with the sum taken over all distinct $r$-tuples $\left(k_{1}, \ldots, k_{r}\right)$ with $k_{j} \in\{1, \ldots, d\}$.

We consider the effect of a general term in (8.6) on $w_{0}$. Since all the operators $D_{k}, k=1, \ldots, d$, and $T(h)$ commute, we can write such a term as

$$
D_{k_{1}} \ldots D_{k_{r}}=T(\xi) \Delta_{h_{1} e_{1}}^{j} \Delta_{h_{2} e_{2}}^{\lambda_{2}} \ldots \Delta_{h_{d} e_{d}}^{\lambda_{d}},
$$

with $0 \leq \lambda_{2}, \ldots, \lambda_{d}$ and $\lambda_{2}+\cdots+\lambda_{d}=r-j$ and $\xi$ some point in $\mathbb{R}^{d}$. The difference operator $\Delta_{h_{1} e_{1}}^{j}$ acts only with respect to $x_{1}$, and the remaining difference operators in (8.7) applies only to $x_{2}, \ldots, x_{d}$. Hence

$$
D_{k_{1}} \ldots D_{k_{r}}\left(w_{0}\right)=T(\xi) \Delta_{h_{1}}^{j}\left(v_{0}, x_{1}\right) \Delta_{h_{2} e_{2}}^{\lambda_{2}}\left(\phi, x_{2}\right) \ldots \Delta_{h_{d} e_{d}}^{\lambda_{d}}\left(\phi, x_{d}\right) .
$$


Since $\phi \in C^{\infty}$, we have for $\mu:=\min (1, \tau)$ and for a constant $C$ depending only on $d$, $r$ and $\mu$,

$$
\omega_{r}\left(w_{0}, s\right)_{\tau}^{\mu} \leq C \sum_{j=0}^{r}\left[s^{r-j} \omega_{j}\left(v_{0}, s\right)_{\tau}\right]^{\mu}
$$

where for the purposes of this formula and the formulas below we define $\omega_{0}\left(v_{0}, s\right)_{\tau}:=$ $\left\|v_{0}\right\|_{L_{\tau}(\mathbb{R})}$ for all $s$. From Marchaud's inequality (see for example $\S 8$ of Chapter 2 in $[5]$ ), the $j$-th term, $j \neq 0, r$, of the sum in (8.8) for $0<s \leq 1$ can be bounded by

$$
C s^{r \mu} \int_{s}^{\infty}\left[\sigma^{-j} \omega_{r}\left(v_{0}, \sigma\right)_{\tau}\right]^{\mu} \frac{d \sigma}{\sigma} \leq C s^{r \mu} \int_{s}^{1}\left[\sigma^{-j} \omega_{r}\left(v_{0}, \sigma\right)_{\tau}\right]^{\mu} \frac{d \sigma}{\sigma}+C s^{r \mu}\left\|v_{0}\right\|_{L_{\tau}(\mathbb{R})}^{\mu} .
$$

Returning to (8.8), we obtain for $s \leq 1$,

$$
\begin{aligned}
\omega_{r}\left(w_{0}, s\right)_{\tau}^{\mu} \leq C s^{r \mu}\left\|v_{0}\right\|_{L_{\tau}(\mathbb{R})}^{\mu} & +\omega_{r}\left(v_{0}, s\right)_{\tau}^{\mu} \\
& +C s^{r \mu} \sum_{j=1}^{r-1} \int_{s}^{\infty}\left[\sigma^{-j} \chi_{[0,1]}(\sigma) \omega_{r}\left(v_{0}, \sigma\right)_{\tau}\right]^{\mu} \frac{d \sigma}{\sigma}
\end{aligned}
$$

It follows therefore from Hardy's inequality (see, e.g., $§ 3$ of Chapter 2 of [5]) that

$$
\begin{aligned}
& \int_{0}^{\infty}\left[s^{-\alpha} \chi_{[0,1]}(s) \omega_{r}\left(w_{0}, s\right)_{\tau}\right]^{\tau} \frac{d s}{s} \\
& \leq C\left\{\left\|v_{0}\right\|_{L_{\tau}(\mathbb{R})}^{\tau}+\sum_{j=1}^{r} \int_{0}^{\infty}\left[s^{r-j-\alpha} \chi_{[0,1]}(s) \omega_{r}\left(v_{0}, s\right)_{\tau}\right]^{\tau} \frac{d s}{s}\right\} .
\end{aligned}
$$

Since $0 \leq s \leq 1$, the terms $s^{r-j-\alpha}$ can each be replaced by $s^{-\alpha}$. We have remarked earlier that in the definition of the Besov norm the integral in (3.2) can be taken over $[0,1]$. Since $v_{0}$ is in $B_{\tau}^{\alpha}\left(L_{\tau}(\mathbb{R})\right)$, we conclude that the right side of $(8.10)$ is finite, and therefore $w_{0}$ is in $B_{\tau}^{\alpha}\left(L_{\tau}\left(\mathbb{R}^{d}\right)\right)$.

(iii) $\tau \geq 1 / \alpha$.

This case can be proved in a similar way to (ii). We let $\tilde{v}$ be the solution to the univariate problem (1.1) with transport velocity $a_{1}$ for a compactly supported univariate function $\tilde{v}_{0}$ in $C^{\infty}$. We can choose $\tilde{v}_{0}$ so that no characteristics meet before time $t=1$ and at time $t=1, \tilde{v}_{1}:=\tilde{v}(\cdot, 1)$ has a single downward jump discontinuity at $x=0$ of size 1 . Moreover, we can require that $\tilde{v}_{1}$ vanishes on $(0, \infty)$ and is continuous on $(-\infty, 0)$.

As in part (ii), we consider the initial condition $w_{0}=\tilde{v}_{0} \varphi$ with $\varphi$ as in (ii). Then $w_{0}$ is in every space $B_{\tau}^{\alpha}\left(L_{\tau}\left(\mathbb{R}^{d}\right)\right)$. At time $t=1$, for $h>0$ sufficiently small, we have

$$
\left|\Delta_{h e_{1}}^{r}\left(w_{1}, x\right)\right| \geq 1 / 2, \quad x=\left(x_{1}, \ldots, x_{d}\right) \in[-1,1]^{d}, \quad-h \leq x_{1} \leq 0 .
$$

Therefore,

$$
\omega_{r}\left(w_{1}, s\right)_{\tau}^{\tau} \geq C s, \quad s \in[0,1]
$$

This gives that

$$
\left|w_{1}\right|_{B_{\tau}^{\alpha}\left(L_{\tau}\left(\mathbb{R}^{d}\right)\right)}^{\tau} \geq \int_{0}^{1} s^{-\alpha \tau} d s
$$

Since $\alpha \tau \geq 1$, the last integral diverges and shows that $w_{1}$ is not in $B_{\tau}^{\alpha}\left(L_{\tau}\left(\mathbb{R}^{d}\right)\right)$. 


\section{REFERENCES}

[1] R. Sharpley and C. Bennett, Interpolation of Operators, Academic Press, New York, N.Y., 1988.

[2] A. P. Calderón, Spaces between $L^{1}$ and $L^{\infty}$ and the theorem of Marcinkiewicz, Studia Mathematica, 26 (1966), pp. 273-299.

[3] M. Crandall and L. TaRtaR, Some relations between nonexpansive and order preserving mappings, Proc. Amer. Math. Soc., 78 (1980), pp. 1-21.

[4] R. DeVore, B. Jawerth, And V. Popov, Compression of wavelet decompositions, Amer. Jour. Math., 114 (1992), pp. 737-785.

[5] R. DeVore and G. G. Lorentz, Constructive Approximation, Grundlehren, Springer Verlag, New York, N.Y., 1993.

[6] R. DeVore and B. Lucier, High order regularity for conservation laws, Indiana Univ. Math. J., 39 (1990), pp. 413-430.

[7] High order regularity for solutions of the inviscid Burgers equation, in Nonlinear Hyperbolic Problems, Springer Lecture Notes in Mathematics 1402, Proceedings of the Advanced Workshop held in Bordeaux, France, June, 13-17,1988, C. Carrasso, P. Charrier, B. Hanouzet, J-L. Joly, eds., Springer Verlag, New York, 1989, pp. 406-413.

[8] R. DeVore and V. Popov, Interpolation of Besov spaces, Trans. Amer. Math. Soc., 305 (1988), pp. 397-414.

[9] - Interpolation spaces and non-linear approximation, in Function Spaces and Applications, Springer Lecture Notes in Math. 1302, M. Cwikel, J. Peetre, Y. Sagher, and H. Wallin, eds., Springer, Berlin, 1988, pp. 191-205.

[10] S. N. KRužKov, First order quasilinear equations in several independent variables, Math. USSR Sbornik, 10 (1970), pp. 217-243.

[11] P. D. LAx, Hyperbolic Systems of Conservation Laws and the Mathematical Theory of Shock waves, Regional Conference Series in Applied Mathematics 11, SIAM, Philadelphia, 1973.

[12] B. J. LuCIER, A moving mesh numerical method for hyperbolic conservation laws, Math. Comp., 46 (1986), pp. 59-69.

[13] _ Regularity through approximation for scalar conservation laws, SIAM J. Math. Anal., 19 (1988), pp. $763-773$.

[14] P. Petrushev, Direct and converse theorems for spline and rational approximation and Besov spaces, in Function Spaces and Applications, Springer Lecture Notes in Mathematics 1302, M. Cwikel, J. Peetre, Y. Sagher, and H. Wallin, eds., Springer, Berlin, 1988, pp. 363-377.

[15] A. I. VOL'PERT, The spaces BV and quasilinear equations, Math. USSR Sbornik, 2 (1967), pp. 225-267. 